\title{
Cluster Crystals in Confinement
}

\author{
Sven van Teeffelen, ${ }^{1, \text { f Angel J. Moreno, }}{ }^{2}$ and Christos N. Likos ${ }^{1,3}$ \\ ${ }^{1}$ Institut für Theoretische Physik II: Weiche Materie, \\ Heinrich-Heine-Universität Düsseldorf, Universitätsstraße 1, D-40225 Düsseldorf, Germany \\ ${ }^{2}$ Centro de Física de Materiales (CSIC-UPV/EHU), Apartado 1072, 20080 San Sebastián, Spain \\ ${ }^{3}$ The Erwin Schrödinger International Institute for Mathematical Physics (ESI), Boltzmanngasse 9, A-1090 Vienna, Austria
}

(Dated: August 9, 2008)

\begin{abstract}
A large class of fluids of particles interacting via ultrasoft, repulsive pair potentials crystallize into cluster crystals. Here, we employ density functional theory and computer simulations to study the behavior of a system of particles that repel each other with a $\exp \left(-r^{8}\right)$-potential [A. J. Moreno and C. N. Likos, Phys. Rev. Lett., 2007, 99, 107801] under planar confinement. We compare the behavior for purely repulsive to that for attractive slit walls. In particular, we present the phase diagram and we show that for repulsive walls the system freezes from the middle, whereas for attractive ones crystallization sets in at the walls and proceeds to the middle. For large wallwall-separations we find continuous growth of a fluid or crystalline layer on the wall, depending on the wall-particle interaction, which is interrupted by capillary melting or freezing close to the bulk crystallization transition. An asymptotic scaling analysis of the width of the liquid or crystalline films growing at the walls indicate complete wetting in both cases.
\end{abstract}

PACS numbers: 64.70.Dv, 82.30.Nr, 61.20.Ja, 82.70.Dd

\section{INTRODUCTION}

The influence of slit pore confinement and of single planar walls on freezing and melting has been studied for many different atomic and colloidal systems [1, 2, 3, 4, by means of computer simulation [5, 6, 7, 8, 9, 10, 11], theory [12, 13, 14, and experiment [3, 15, 16. In general, two limiting cases of planar confinement are to be distinguished in terms of the pore size: In narrow pores of the width of one or few particle diameters, crystallization of the quasi-2D system is strongly influenced by the wall-particle interaction and by packing effects [8, 17, 18, 19]. For monolayers or even a few hard-sphere layers in planar confinement, the otherwise first-order Kosterlitz-Thouless phase transition becomes continuous [20, 21. On the other hand, for large confinement widths, freezing and melting is dominated by the $3 \mathrm{D}$ bulk phase behavior and by single-wall properties, i.e., crystal/fluid wetting [6, 7, 9, For large confinement widths, two possible scenarios are observed upon approaching the bulk liquid-solid transition, depending on the different interactions between the wall, the liquid, and the crystal: In the first scenario, the walls induce melting, which becomes manifest in a fluid layer growing on the walls, already under conditions in which the crystal is stable in the bulk, a situation referred to as premelting 12, 13, 14, 15. In the second scenario, the walls induce freezing, which is due to the presence of a crystalline slab under conditions in which the fluid is stable in the bulk, denoted as prefreezing [5, 6, 7, 8, 9]. The former mechanism is responsible for surface melting, which prevents the persistence of overheated crystalline

*teeffelen@thphy.uni-duesseldorf.de states 15. On the other hand, the latter mechanism prevents undercooling of the fluid state; after a quench from a high to a low temperature it is responsible for heterogeneous nucleation on the walls [22] which is often much more likely than homogeneous nucleation deep in the (bulk) fluid state.

Despite a host of literature on experiments, simulation studies, and phenomenological theories, there have been only few attempts to study confinement-induced freezing or melting by means of microscopic theories [1], in which the only input should be the particle-particle and the wall-particle interaction potentials. The method of choice is, evidently, classical density functional theory (DFT) [4, 23, 24, 25, 26]. DFT is an exact reformulation of the statistical mechanics of many-body systems, uniform and nonuniform alike, based on the equilibrium one-particle density $\rho_{\text {eq }}(\mathbf{r})$ of the system. As such, it treats fluids ( $\rho_{\text {eq }}(\mathbf{r})=\rho$, a prescribed constant $)$ and crystals $\left(\rho_{\text {eq }}(\mathbf{r})\right.$ is a periodically modulated space field) on equal footing. In fact, several of the commonly used liquid-state theories can be derived from a DFT formulation [27, whereas the DFT-treatment of crystals can be traced back to the pioneering work of Ramakrishnan and Yussouff [28]. Concomitantly, DFT offers also a tool to study bulk phase coexistence, a prerequisite for the subsequent investigations of fluid-solid interfaces 29]. There have been DFT calculations for surface melting of Lennard-Jones particles [12, 13, for hard spheres on hard walls [13, for hard spheres in very thin slit pores 30, and also for hard spheres sedimenting onto hard walls 31. Hard spheres in slit pores have been examined by means of macroscopic, thermodynamicallyinspired arguments 32 as well as by computer simulations in a system similar to the one that is the subject of this work, in the sense that it also forms clusters, albeit of a different nature than the ones we consider here [33]. 
Quite a bit of theoretical work and simulation studies have been carried out for liquid-gas separation and wetting phenomena of fluids in confinement [24, 34. However, to the best of our knowledge, the effect of varying confinement-width on crystallization has not been systematically studied by means of DFT to-date.

In this work, we study the influence of planar confinement on a special class of colloidal particles, which interact via bounded and purely repulsive pairwise additive potentials. In particular, these pair potentials do not diverge at zero particle distance. These so called ultrasoft, repulsive particles are realized in nature and experiment by a large class of complex molecules, such as branched or non-branched polymers that possess a vast number of internal degrees of freedom, a property which allows them to share their (center-of-mass) position with another particle or even several other particles of their kind 35. The effective interaction of such complex molecules is highly tunable by choosing different architectures and solvabilities of the respective polymer monomers, model amphiphilic dendrimers being a concrete example 36. Recent interest in such potentials has grown not only on the basis of their physical relevance but also due to some beautiful mathematical properties they possess, allowing for the determination of exact ground states [37, 38] and the application of generalized duality relations to such systems 39.

The rest of this work is organized as follows: In Section III we introduce the model interaction and briefly review its properties. In Section III we give a short description of the DFT, whereas in Section [IV we describe the numerical simulation technique used. The bulk phase behavior is discussed in Section $\mathrm{V}$. The system confined between repulsive walls is studied in Section VI, whilst the attractive walls are studied in Section VII Finally, in Section VIII we summarize and draw our conclusions.

\section{THE MODEL: ULTRASOFT, REPULSIVE PARTICLES}

The particles considered here interact by means of a non-negative and bounded pair interaction potential, $0 \leq \phi(r)<\infty$, that is integrable and possesses a Fourier transform $\tilde{\phi}(k)$, and are termed ultrasoft, repulsive particles. Such interactions have been divided into two categories, the so called $Q^{ \pm}$- and the $Q^{+}$-class [40]. The former classifies potentials for which $\tilde{\phi}(k)$ has an oscillatory decay about zero, while interaction potentials in systems of the latter kind fulfill the condition $\tilde{\phi}(k) \geq 0$. The drosophila of bounded pair potentials is the generalized exponential model of index $n$ (GEM- $n$ ), which is defined by

$$
\phi(r)=\epsilon \exp \left[-(r / \sigma)^{n}\right],
$$

where $r$ is the particle distance, $\sigma$ is the interaction range (typically a measure of the particle's size), and $\epsilon$ is the interaction strength. In the following, we will set $\epsilon=1$, $\sigma=1$ as well as $k_{\mathrm{B}}=1$, the latter being Boltzmann's constant. It has been shown [41] that $\phi(r)$ of eq. (1) belongs to the $Q^{+}$-class for $n \leq 2$ and to the $Q^{ \pm}$-class for $n>2$. Moreover, it has been established [40] that the distinction of $Q^{ \pm}$- and $Q^{+}$-particles goes along with two different principle features of the topologies of the respective bulk phase diagrams: Systems of the $Q^{ \pm}$-kind display freezing for arbitrarily high temperatures and densities into so called cluster crystals [36, 41, 42, 43, while systems of the $Q^{+}$-kind are characterized by reentrantmelting and a maximum freezing temperature [44. In this paper, we concentrate on the class of $Q^{ \pm}$-particles and their behavior in confinement.

Cluster crystallization is highly distinct from crystallization of "conventional/ordinary" colloids: It implies that at high densities several particles share the same lattice site of a periodic crystal. In fact, it has been shown for the GEM-4 model that upon increasing the density, the lattice constant of the stable fcc crystal, $a=\sqrt{2} d$, where $d$ is the nearest-neighbor distance, approaches a density- and temperature-independent constant [36. The above implies that the average number of particles sharing a lattice site, $n_{c}$, grows linearly with density. In this work, we investigate the phase behavior of GEM-8-particles, i.e., representative ones of the $Q^{ \pm}$-species, which show a tendency to cluster at a lower density than the GEM-4-particles do, due to a deeper minimum of the respective Fourier-transformed pair potential [41. Freezing is studied with the help of mean field density functional theory (MFA-DFT) and with simulation for different wall-particle interactions and for different wall-wall separation $L_{z}$. MFA-DFT has proved to be highly accurate to reproduce the bulk phase behavior of the GEM-4 model [43, and will be shown here to accurately predict the equilibrium phase diagram of the GEM-8 in the bulk and in confinement.

The main focus of this work is in the behavior of the GEM-8 model in slit pores. Two kinds of planar confinement are considered: In a first setup we study the effect of purely repulsive Yukawa-walls on the structure and phase diagram of the system while in a second setup the influence of attractive Lennard-Jones walls is examined. The Yukawa potential has been shown to model the interaction of dendrimers with hard walls [45, 46]. In order to model attractive walls we introduce a 9-3-LennardJones potential. The latter interaction is not based on microscopic grounds but is introduced to model the influence of attractive wall potentials in general. However, recent simulation results [46] have shown that dendrimers on walls with core-monomer-wall attractions show very similar effective interaction potentials. We demonstrate that the first setup leads to premelting: the system is molten at the walls and crystallizes at the center, while for the second setup the opposite happens, i.e., there the walls induce prefreezing [1, 2]. Furthermore, we argue that both setups display complete wetting [47] which is deduced from a continuous, logarithmic growth of the fluid/crystalline wetting layer down/up to the point of 
capillary melting/freezing.

A salient property that distinguishes the behavior of clustering particles from non-clustering ones in confinement, is the ability to locally adjust the average occupation number of the clusters, $n_{c}$, without distorting the crystal structure through point defects such as vacancies or interstitials. Remarkably, although there is incessant hopping of particles from one site to the other, rendering these crystals ergodic and endowing them with a non-vanishing long-time diffusivity, the underlying crystal structure remains intact. In fact, this happens not despite the hopping mechanism but because of it. For details, see Ref. [48, 49].

\section{DENSITY FUNCTIONAL THEORY}

Density functional theory is based on a variational grand canonical functional $\tilde{\Omega}[\rho(\mathbf{r})]$, a unique functional of the static one-particle density $\rho(\mathbf{r})=\left\langle\sum_{i=1}^{N} \delta\left(\mathbf{r}-\mathbf{r}_{i}\right)\right\rangle$ of the system, where $\mathbf{r}_{i}, i=1 \ldots N$ are the particle coordinates and $\langle\ldots\rangle$ denotes the according grand canonical average 23 . The functional $\tilde{\Omega}[\rho(\mathbf{r})]$ is minimized by the equilibrium one-particle density $\rho_{\text {eq }}(\mathbf{r})$, where it takes the value of the system's grand potential, $\Omega[\rho(\mathbf{r})]$. The density functional is typically split into the ideal gas, an excess, and an external part,

$$
\begin{aligned}
\tilde{\Omega}[\rho(\mathbf{r})] & =F_{\text {id }}[\rho(\mathbf{r})]+F_{\text {ex }}[\rho(\mathbf{r})] \\
& +\int \mathrm{d} \mathbf{r} \rho(\mathbf{r})(V(\mathbf{r})-\mu),
\end{aligned}
$$

where $V(\mathbf{r})$ is the external potential and $\mu$ is the chemical potential. The integral runs over the system volume. The ideal part reads as

$$
F_{\text {id }}[\rho(\mathbf{r})]=\beta^{-1} \int \mathrm{d} \mathbf{r} \rho(\mathbf{r})[\ln \rho(\mathbf{r})-1]+3\langle N\rangle \beta^{-1} \ln \Lambda,
$$

with $\beta^{-1}=T,\langle N\rangle=\int \mathrm{d} \mathbf{r} \rho(\mathbf{r})$ being the average particle number and $\Lambda$ the thermal de Broglie wavelength. The last term in eq. (3) above is thermodynamically irrelevant and will be ignored henceforth. The excess part is given very accurately for sufficiently high temperatures and/or densities by the mean-field expression [4]

$$
F_{\text {ex }}[\rho(\mathbf{r})]=\frac{1}{2} \iint \mathrm{d} \mathbf{r} \mathrm{d} \mathbf{r}^{\prime} \rho(\mathbf{r}) \rho\left(\mathbf{r}^{\prime}\right) \phi\left(\mathbf{r}-\mathbf{r}^{\prime}\right) .
$$

It is pertinent, at this point, to shortly comment on the accuracy of the expression (4) above. In Ref. 41, it has been shown that this functional form rests on the validity of the approximation $c(r)=-\beta \phi(r)$ for the direct correlation function $c(r)$ of the fluid (uniform) phase. Within the framework of linear response theory and employing the Percus identity, it can be shown that the relation $c(r)=-\beta \phi(r)$ holds asymptotically in regions of space for which the potential $\phi(r)$ caused by a test particle held fixed at the origin is much weaker than the other two energy scales of the problem: the thermal energy $T$ and/or the average potential energy per particle caused by all other particles in a fluid of density $\rho$ [27. For diverging potentials, this relationship must break down at sufficiently small $r$-values [50. However, for ultrasoft, bounded potentials, it can and it indeed does hold approximately true for all separations $r$ provided $T$ and/or $\rho$ are sufficiently high, so that the thermal and/or the potential energy per particle dominate over $\phi(r)$. In previous work [40, 41] it was demonstrated that the conditions $T \gtrsim 1$ and/or $\rho \gtrsim 1$ are sufficient for the relationship $c(r)=-\beta v(r)$ to be fulfilled to a satisfactory degree of approximation.

Minimization of $\tilde{\Omega}[\rho(\mathbf{r})]$ is pursued by demanding its functional derivative with respect to density to vanish. This amounts to numerically solving the self-consistent equation

$\rho_{\mathrm{eq}}(\mathbf{r})=\exp \left\{\beta \mu-\beta V(\mathbf{r})-\int \mathrm{d} \mathbf{r}^{\prime} \beta \phi\left(\left|\mathbf{r}-\mathbf{r}^{\prime}\right|\right) \rho_{\mathrm{eq}}\left(\mathbf{r}^{\prime}\right)\right\}$,

for $\rho_{\text {eq }}(\mathbf{r})$ in an iterative fashion, on a periodic rectangular grid of $N_{x} \times N_{y} \times N_{z}$ grid points, where the convolution integral is evaluated with the help of fast Fourier transform. We chose a grid of $N_{\alpha} \approx 16 L_{\alpha}, \alpha=x, y, z$ which is fine enough to reach numerical convergence for all state points under study. For the study of crystalline states the $x$ - and $y$-dimensions of the box are chosen to be commensurable with the expected lattice spacings in plane with the confining walls (see Section $\mathrm{V}$ ). The lattice spacing of crystalline GEMs in the bulk is well known to be relatively insensitive towards density/chemical potential and temperature. This is also found for the GEM- 8 in confinement, as will be shown below.

\section{SIMULATION}

We simulated a system of several thousand particles, $N$, interacting through a GEM- 8 potential $\phi(r)=$ $\exp \left(-r^{8}\right)$. For the case of confinement, they also experience an external wall potential $V(z)$ presented in Sections VI and VII. Simulation results presented here are obtained by means of Brownian dynamics. The equation of motion for the position vector $\mathbf{r}_{i}$ of a particle $i$ is given by:

$$
\dot{\mathbf{r}}_{i}(t)=-\Gamma \nabla_{\mathbf{r}_{i}}\left[V\left(\mathbf{r}_{i}\right)+\sum_{j \neq i} \phi\left(\left|\mathbf{r}_{i}-\mathbf{r}_{j}\right|\right)\right]+\mathbf{w}_{i}(t),
$$

where $\Gamma$ is a mobility constant (we set $\Gamma=1$ ) and $\mathbf{w}_{i}(t)$ is a stochastic Gaussian noise term. The latter represents the random collisions with the much faster solvent molecules, which are not explicitly included in the model. The noise fulfills the statistical properties [51]

$$
\left\langle\mathbf{w}_{i}(t)\right\rangle=0, \quad\left\langle w_{i}^{\alpha}(t) w_{j}^{\beta}\left(t^{\prime}\right)\right\rangle=2 \beta^{-1} \delta_{\alpha \beta} \delta_{i j} \delta\left(t-t^{\prime}\right),
$$


where $\alpha, \beta$ are the Cartesian $x, y, z$-components.

We implemented periodic boundary conditions for the simulation cell of volume $V_{c}=L_{x} \times L_{y} \times L_{z}$. Periodicity was applied in the $x, y$-directions in all cases, and also in the $z$-direction for the bulk case. We employed a cubic cell of size $L_{x}=L_{y}=L_{z}=18.57$ for the bulk system. In the confined system, we set $L_{x}=L_{y}=20.43$ for slits of thickness $L_{z} \leq 8$. We also carried out simulations on the confined system for thickness $L_{z}=24$, with $L_{x}=L_{y}=$ 9.285 .

The Brownian dynamics simulations were performed at constant $N, V_{c}$, and $T$, and the equations of motion (6) were integrated via the Ermak's algorithm [51, 52, with a timestep $\Delta \tau=10^{-4} \tau_{B}$. Here, $\tau_{B}=\beta$ is the Brownian time scale. The system was prepared by randomly placing the particles in the simulation cell. In this way, crystallization occurs spontaneously at sufficiently high density. An equilibration run of typically $10^{6}$ timesteps was performed before the production run of typically $3 \times 10^{6}$ timesteps. During the production run, configurations were periodically saved for computation of observables. Density profiles and radial distribution functions (see below) were averaged over typically 30 configurations, the interval between two consecutive configurations of the latter being 100000 timesteps. In this time scale, the particle mean displacement is of at least one molecular diameter for all the investigated systems, guaranteeing that the former configurations are uncorrelated. At high densities, a few independent runs were also performed for some fixed state points $\left(\rho, T, L_{z}\right)$, starting from different configurations of the particles, in order to check that the final values of the former observables are independent of the initial conditions.

\section{THE BULK PHASE DIAGRAM}

The phase behavior in confinement is determined by two factors, the interaction of the particles with the walls and the behavior in the bulk; in particular, the location of the considered thermodynamic point $(\mu, T)$ with respect to bulk phase boundaries plays a decisive role in influencing the density profiles in confinement and related surface phase transitions. Before presenting the phase diagram of the system in confinement we therefore provide the bulk phase behavior, as obtained from the DFT, which serves as a reference point.

Solving eq. (5) within the minimum rectangular unit cell of the body centered cubic (bcc), the face centered cubic (fcc) and the hexagonal close packed (hcp) lattices of variable lattice spacing, we find that for temperatures $0.5 \leq T \leq 4$ the system undergoes two subsequent firstorder transitions upon increasing the chemical potential $\mu$, first from the liquid to a hcp cluster crystal at $\mu_{f}$, and at higher $\mu$, a subsequent structural phase change from a hcp to a fcc cluster crystal. This scenario is in contrast to other GEM- $n$ models with smaller $n$ where at first a postfreezing bcc cluster phase is found, preceding a bcc $\rightarrow$ fcc transformation at higher values of $\rho($ or $\mu)$ [42, 43]. We have not carried out a search of other non-Bravais lattices than the hcp-one. Thus it cannot be ruled out that there are other stable phases of non-Bravais lattices at freezing or at higher $\mu$. However, we can clearly state that within the DFT the post-freezing lattice is not a Bravais lattice.

For $T=1$, which is the temperature for all confinement studies throughout this work, we locate the freezing transition in the bulk at a chemical potential $\mu_{f}=12.09$. Here, a fluid of density $\rho_{f}=2.96$ coexists with the hcp cluster crystal of density $\rho_{s}=3.48$. The latter crystal consists of polydisperse clusters that contain on average $n_{c} \cong 6.02$ particles each, corresponding to an equilibrium nearest neighbor distance of $d=2^{1 / 6}\left(n_{c} / \rho_{s}\right)^{1 / 3} \cong 1.347$. The second transition towards the fcc crystal takes place at a chemical potential $\mu=20.03$. Here, already deep in the crystalline phase, an hcp lattice of average density $\rho=7.80$, average occupation number $n_{c}=11.96$, and nearest neighbor distance $d=1.295$ coexists with a fcc lattice of average density $\rho=7.82$, occupation number $n_{c}=11.85$, and nearest-neighbor distance $d=1.289$ implying a lattice constant of $a=\sqrt{2} d=1.823$. We anticipate that, similar to the GEM-4-model, there is a triple point at which liquid, hcp crystal and fcc crystal coexist.

The detailed investigation of the bulk phase behavior of the GEM-8-model is not the purpose of this work, yet a few comments are of order. First, the stability of the hcp lattice with respect to the fcc comes as a surprise, since the latter features a larger distance to the thirdnearest neighbors of a given particle than the first. On energetic grounds, one would thus expect the fcc to win, and it indeed does so at $T=0$. But very much like the bcc-lattice is the post-freezing structure above the triple point of the GEM-4-model on entropy grounds, the hcp achieves a lower free energy than the fcc due to an entropically more favorable arrangement of the density profiles around the crystal sites 53. As density grows, however, so does also the cluster population $n_{c}$. The inter-site interaction energy, which scales as $n_{c}^{2}$, becomes increasingly important, energy takes over and fcc wins over hcp. There are indications from the simulations in confinement that at post-freezing densities the ordered state indeed has the propensity to undertake a hcp-like ordering, i.e., an $A B A B$.. stacking of hexagonal arrays, as opposed to the $A B C A B C \ldots$ stacking of the fcc, as shown in fig. 1. However, in bulk simulations the system spontaneously forms a bcc lattice at freezing. The determination of accurate phase boundaries and crystal phases could be pursued by means of the novel simulation technique of Mladek, et al. 36. This task goes beyond the scope of this work and will be the subject of further investigations.

For the rest of this paper, we sidestep thus the issue of the stability of the hcp-lattice and the associated question of the possible existence of other non-Bravais lattices and restrict our theoretical studies to Bravais crystals only. The reasons for doing this are twofold: first, 

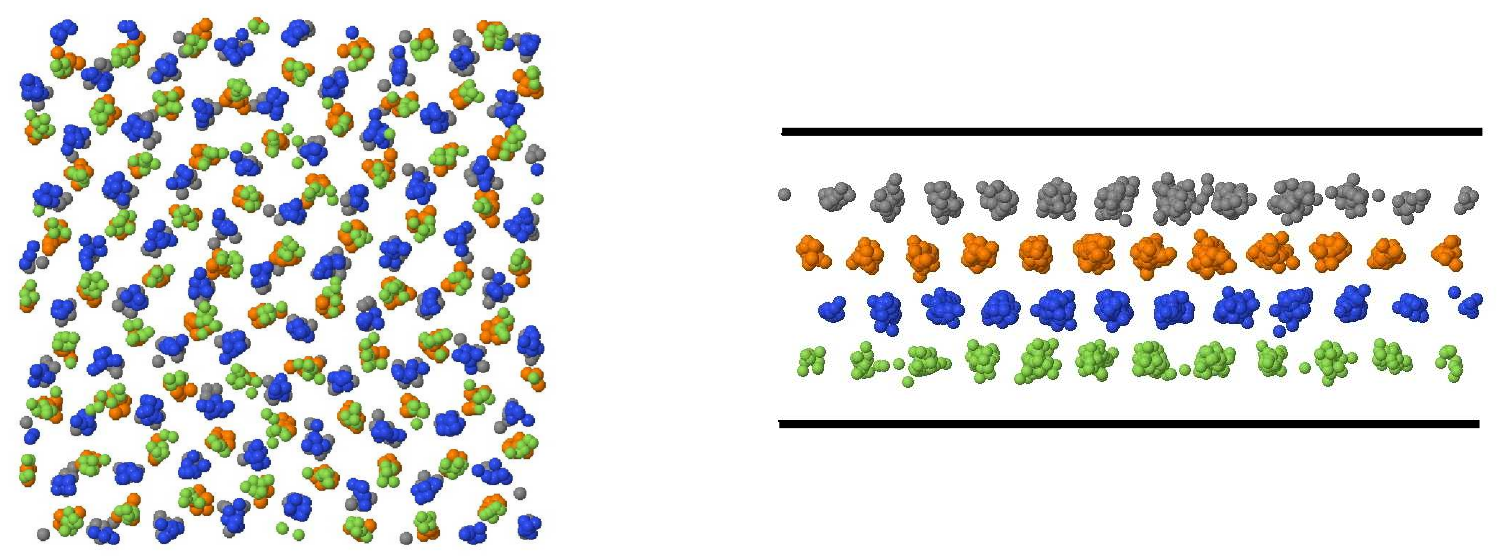

FIG. 1: Left panel: simulation snapshot of the four crystalline layers of a confined GEM-8-model at temperature $T=1$ and density $\rho=4.2$, view of one quarter of the $x y$-periodic box from the bottom (lower wall). The two repulsive walls have a separation $L_{z}=5.2$ and are lying parallel to the page. The layers of clusters are color-coded, from bottom to top: green, blue, orange, and gray. An $A B A B \ldots$ stacking of the hexagonal layers can be seen. Right panel: same as the left but now from the side view.

as mentioned above, because it is not clear which other non-Bravais lattices might be competitive. Second, we expect that our results are generic, since the physics is dictated by the tendency of the model to form cluster crystals and is not dependent on the detailed spatial arrangement of the same on a specific lattice. Therefore, we focus on the bulk results for the phase boundary between a liquid and a fcc crystal, ignoring any other candidate periodic arrangement; among all Bravais lattices, the fcc lattice is the most stable one. The bulk freezing transition for the same temperature $T=1$ within the DFT is found at a slightly higher chemical potential than for the liquid-hcp transition, namely $\mu_{f}=12.13$. Here, a fluid of density $\rho_{f}=2.97$ coexists with the fcc cluster crystal of density $\rho_{s}=3.50$. The fcc crystal consists of polydisperse clusters that contain on average $n_{c} \cong 6.04$ particles each, corresponding to an equilibrium lattice constant of $a=\left(4 n_{c} / \rho_{s}\right)^{1 / 3} \cong 1.905$. The equilibrium lattice constant exceeds the value

$$
a^{*}=2 \sqrt{3} \pi / k^{*}=1.859
$$

where $k^{*}=5.855$ is the wavenumber for which $\tilde{\phi}(k)$ attains its minimum, negative value, by a factor 1.025 . The lattice constant $a^{*}$ corresponds to a nearest-neighbor distance $d^{*}=2^{-1 / 2} a^{*}=1.314$ and to a distance of adjacent layers of hexagonally crystalline particles in the 111-direction of the fcc crystal $c^{*}=a^{*} / \sqrt{3}=1.073$ [54]. The result of eq. (8) stems from a simplified version of the MFA-DFT, which takes only the first reciprocal lattice vector (RLV) of the fcc lattice into account and results into the outcome that the length of the first shell of RLVs of the fcc crystal should coincide with $k^{*}$ at all densities [1]. The equilibrium lattice constant of the

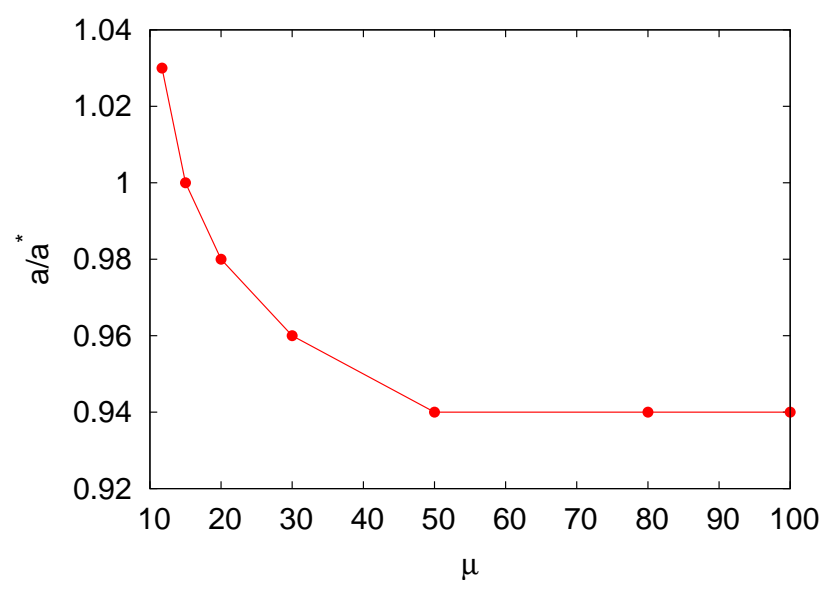

FIG. 2: The equilibrium lattice constant for the fcc crystal in the bulk as a function of chemical potential for $T=1$.

fcc crystal resulting from the full DFT-minimization is relatively insensitive towards temperature changes. Furthermore, as is known for the GEM-4 as well, it is almost independent of $\mu$. In particular, for $T=1, a$ decays as a function of $\mu$ towards a plateau of $a=0.94 a^{*}$ which it reaches at $\mu \sim 50$, as can be seen in fig. 2 .

In view of the aforementioned insensitivity of $a$ with respect to the state point in the phase diagram, we can simplify the calculation by fixing it to a constant value $a=a^{*}$ for all state points $(\mu, T)$ and recalculate the bulk phase diagram under this constraint. Thereby, a slightly higher chemical potential at freezing is obtained: For $T=$ 1 , the value $\mu_{f}=12.25$, with coexistence densities of 


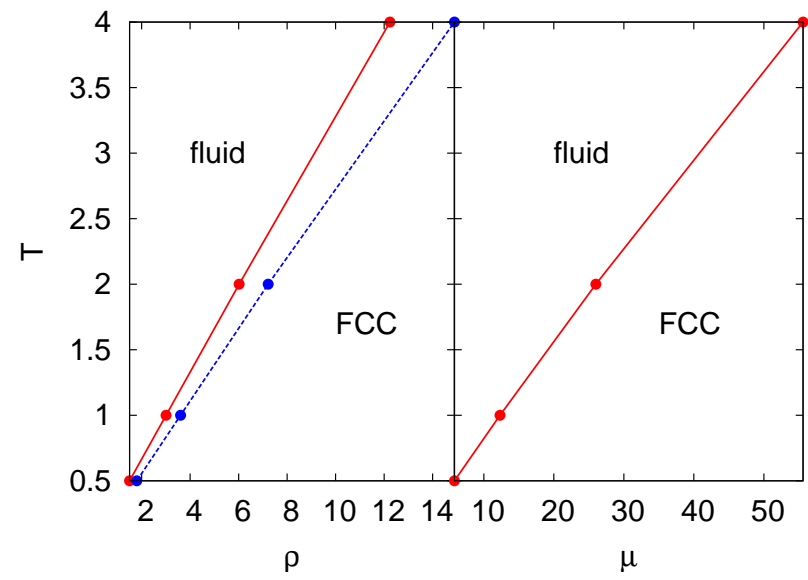

FIG. 3: The (constrained) bulk phase diagram for fixed lattice constant $a^{*}$ and for the fixed fcc-lattice. Left panel: The phase diagram at the $(\rho, T)$-representation. The lines are the loci of the coexisting liquid- and fcc-densities and the gap inbetween denotes the coexistence region. Right panel: same as the left one but at the $(\mu, T)$-representation.

$\rho_{f}=3.00$ and $\rho_{s}=3.58$, and average cluster occupancy $n_{c} \cong 5.74$ results. The chemical potential $\mu_{f}$ and the densities of coexistence are plotted versus temperature in fig. 3 [55.

Another constraint to the lattice geometry, which becomes important for the confinement studies, is the following: The distance of neighboring layers of hexagonally crystalline particles in the 111-direction of the fcc crystal $c$ is free to vary whereas the nearest-neighbor distance of particles within each plane is fixed to $d=d^{*}$. If we minimize the grand potential in this partially constrained fashion $\left(d=d^{*}\right.$ only in the $x y$-plane of confinement), we obtain yet a third value for the bulk freezing chemical potential, $\mu_{f}=12.19$, which lies in-between the two bulk values $\mu_{f}=12.13$ (free lattice constant $a$ ) and $\mu_{f}=12.25$ (fixed lattice constant $a=a^{*}$ ). The optimal distance of neighboring layers was then found to be given by $c=1.113$ which exceeds the corresponding distance $c^{*}$ in an isotropic fcc-crystal with lattice constant $a^{*}$ by a factor 1.038. The bulk freezing parameters obtained by DFT under the various constraint conditions on the minimization procedure are summarized in table $\mathbb{I}$.

\section{REPULSIVE SLIT PORES}

In the first setup we study the effect of the repulsive confining walls. The latter are characterized by the external potential

$$
V_{\mathrm{Y}}(\mathbf{r})=10\left[\frac{e^{-z}}{z}+\frac{e^{-\left(L_{z}-z\right)}}{L_{z}-z}\right], \quad 0<z<L_{z},
$$

where $z$ is the component of the particle coordinate $\mathbf{r}$ perpendicular to the wall, measured with the origin on one of the walls. The Yukawa form is motivated on the basis of results for the interactions of athermal dendrimers (GEM-2-particles) with model planar walls [45] and is confirmed by recent results of Lenz, et al. [46]. The prefactor 10 in eq. (9) is so far arbitrary but should roughly scale linearly with the number of monomers of a dendrimer.

In the DFT, the $x y z$-periodic box for the density field has the dimensions of $L_{x} \times L_{y} \times\left(L_{z}+1\right)$, where neighboring slits in the $z$-direction are separated by a large additional barrier of width 1 , which is wide enough to obviate any mutual particle interactions across the wall. The system volume itself is only of size $L_{x} \times L_{y} \times L_{z}$, excluding the barrier. The dimensions parallel to the walls, $L_{x}$ and $L_{y}$, are chosen to be commensurate with the fcclattice of lattice constant $a^{*}$ either at the 100- or the 111direction perpendicular to the walls (100: $L_{x}=L_{y}=a^{*}$, 111: $\left.L_{x}=2^{-1 / 2} a^{*}, L_{y}=\sqrt{3 / 2} a^{*}\right)$. The 110-orientation is unfavorable due to inefficient packing and is therefore not considered. As already mentioned in the determination of the bulk phase diagram, we did not vary the nearest neighbor distance in the $x$ - and $y$-direction in the slit pore geometry. However, we left the system freedom in adjusting its lattice constant in the $z$-direction, i.e., perpendicularly to the walls.

In confinement, freezing is defined at the point in which a fully modulated density field $\rho(\mathbf{r})$ yields a grand potential lower than that of the $z$-modulated fluid with density $\rho(z): \Omega\left[\rho(\mathbf{r}) ; L_{z}\right]<\Omega\left[\rho(z) ; L_{z}\right][56$ ] and it occurs at a corresponding freezing chemical potential in confinement, $\mu_{f}\left(L_{z}\right)$. It will be shortly demonstrated that due to the repulsive nature of the Yukawa walls, the bulk freezing chemical potential $\mu_{f}$ can be independently obtained as $\mu_{f}=\mu_{f}\left(L_{z} \rightarrow \infty\right)$ by an appropriate extrapolation procedure. Since we minimize the grand potential in a partially constrained fashion (fixed $d=d^{*}$ only in the $x y$-plane, which will turn out to be perpendicular to the 111-direction of the equilibrium fcc-crystal in slit pores of large widths), the asymptotic value of $\mu_{f}\left(L_{z} \rightarrow \infty\right)$ should coincide with the bulk freezing chemical potential $\mu_{f}=12.19$ as obtained by applying the partially constrained minimization procedure in Section $\mathrm{V}$ (cf. table I. line 4). The coincidence of the results obtained by two independent routes for the determination of $\mu_{f}$ serves as a confirmation of the validity of the extrapolation procedure to be employed.

In DFT, symmetry does not break spontaneously. To avoid getting trapped in metastable minima of the free energy landscape in our search for the true equilibrium configuration, we iteratively solve eq. (5) starting from different initial density fields. In particular, we start both from a purely $z$-modulated density field, which always leads to a stable or metastable fluid state and from highly modulated fields, which possess the symmetry of the fcc crystal everywhere but very close to the confining walls, trying different orientation and offsets. To be precise, we start from 111- and 100-oriented crystals (with respect to the walls), which have either a particle layer centered 
TABLE I: Freezing parameters for the liquid-solid transition in the bulk obtained within mean field density functional theory for different constraints to the lattice types of the crystalline phase (hcp, fcc, distorted fcc) and for different unit cell dimensions (fixed vs variable spacing of neighboring hexagonal layers $c$ and fixed vs variable nearest-neighbor distance within each plane $d)$. The freezing parameters are the chemical potential $\mu_{f}$, the densities of the coexisting liquid $\left(\rho_{f}\right)$ and solid $\left(\rho_{s}\right)$, the in-plane nearest neighbor distance $d$, the distance of neighboring planes $c$, and the average occupancy $n_{c}$.

\begin{tabular}{llllllll}
\hline \hline lattice & minimization & $\mu_{f}$ & $\rho_{f}$ & $\rho_{s}$ & $d / d^{*}$ & $c / c^{*}$ & $n_{c}$ \\
\hline hcp & free & 12.09 & 2.96 & 3.48 & 1.025 & 1.025 & 6.02 \\
fcc & free $_{\text {fcc }}$ & 12.13 & 2.97 & 3.50 & 1.025 & 1.025 & 6.04 \\
distorted fcc $^{a}$ & constrained $^{a}$ & 12.25 & 3.00 & 3.58 & 1 & 1 & 5.74 \\
\hline \hline
\end{tabular}

\footnotetext{
${ }^{a}$ The (isotropic) lattice constant is held fixed $\left(a=a^{*}\right)$.

${ }^{b}$ The nearest-neighbor distance within a plane of hexagonally crystalline particles is held fixed $\left(d=d^{*}\right)$ but the inter-plane distance $c$ is allowed to relax freely (cf. text).
}

about the middle of the box $\left(z=L_{z} / 2\right)$ or a particle layer shifted by half an inter-plane distance from $z=L_{z} / 2$. The 111-oriented fcc crystal always leads to a stacking of hexagonally ordered clusters in the $A B C A B C$-fashion. The $A B A B$-stacking resulting from an hcp-crystal is not tested within the theory (see the above discussion of the bulk phase behavior).

Due to the slow exponential decay of the wall-particle potential, the clusters in the layers closest to the walls are less populated and blurred compared to the middle of the slit. This can be seen from the plots of the $x$ - and $y$-averaged density field $\rho_{x y}(z) \equiv\left(L_{x} L_{y}\right)^{-1} \iint \mathrm{d} x \mathrm{~d} y \rho(\mathbf{r})$ for different average densities $\rho=L_{z}^{-1} \int \mathrm{d} z \rho_{x y}(z)$ in fig. 4(a), as obtained from the DFT. We therefore introduce the notion that the crystal freezes from the middle under the influence of the repulsive, Yukawa walls. For comparison, we present in fig. 4(b) results from BD computer simulations for the same parameters as in the DFT, which show the same quantitative behavior for $\rho=2$ and the same qualitative behavior for $\rho=4$. For $\rho=3$, the DFT predicts a crystalline state whereas the system remains fluid in the simulation. The difference in the density profiles for the states at $\rho=3,4$ is associated with a difference in the bulk liquid/solid coexistence densities which has already been observed for the GEM-4 model when applying the compressibility route to the Helmholtz free energy [57].

The pronounced crystallinity in the central region of the simulation box can be observed not only in the density profiles but is also confirmed by measurements of the restricted pair-distribution function 1 in the $x y$-plane,

$$
g_{2 \mathrm{D}}^{(k)}\left(r_{\|}\right) \equiv \frac{V_{k}}{N_{k}^{2}}\left\langle\sum_{i, j(i \neq j)}^{\prime} \delta\left(\mathbf{r}_{\|}-\left|\mathbf{r}_{i \|}-\mathbf{r}_{j \|}\right|\right)\right\rangle .
$$

Here, $\mathbf{r}_{\|}$denotes the lateral projection (onto the slit plane $)$, i.e., $\mathbf{r}_{\|} \equiv \mathbf{r}-\left(\mathbf{r} \cdot \hat{\mathbf{e}}_{z}\right) \hat{\mathbf{e}}_{z}$, where $\hat{\mathbf{e}}_{z}$ is the unit vector in the $z$-direction. The double sum in eq. 10 runs over all $N_{k}$ particles $i$ and $j$ lying in the $k$ th layer from the wall (this restriction being denoted by the prime) with volume $V_{k}$. Particles belong to the same layer if their position lies between the same two consecutive minima of the respective laterally-averaged density profile $\rho_{x y}(z)$. We show $g_{2 \mathrm{D}}^{(k)}\left(r_{\|}\right)$for the first three layers from either wall, $k=1,2,3$, in fig. 5. Peaks and minima of the pair distribution function clearly become more pronounced for increasing distance of the layer from the wall. Also, the appearance of a shoulder at the third peak of $g_{2 \mathrm{D}}^{(2)}$ and $g_{2 \mathrm{D}}^{(3)}$ indicates the strong crystallinity of the clusters within the respective layers.

It is important to note that the ability of the particles to form clusters has two profound consequences for the interpretation of the density field $\rho(\mathbf{r})$, qualitatively distinguishing them from ordinary colloidal crystals: First, the decreased sharpness of the density peaks close to the walls as compared to the central region of the slit is not only to be interpreted as a fluctuation of the clusters' center-of-mass position but also and rather as an increase of the cluster radii, i.e., an increase of the mean distance of the particles from their cluster's center of mass. Second, as was already mentioned in the introduction, the different average numbers of particles occupying the cluster peaks indicated by the numbers in fig. 4, do not go along with distortions of the lattice structure. In contrast to freezing of usual colloidal systems in confinement, even for the smallest average densities above freezing the average number of particles occupying a cluster is substantially larger than 1 . In fact, it is these features which make the system very amenable to a mean field DFTtreatment.

For all confinement lengths $2 \leq L_{z} \leq 48$ studied, the freezing chemical potential in confinement is higher than in the bulk, $\mu_{f}\left(L_{z}\right)>\mu_{f}$, see fig. 6. freezing is hindered by the confining Yukawa walls. On the other hand, the fluid and the fcc solid average densities $\rho_{f}\left(L_{z}\right)$ and $\rho_{s}\left(L_{z}\right)$ at coexistence are smaller than the respective bulk values for $L_{z} \gtrsim 1$, as can be seen in fig. 7 . The reason lies in the decreased probability density to 

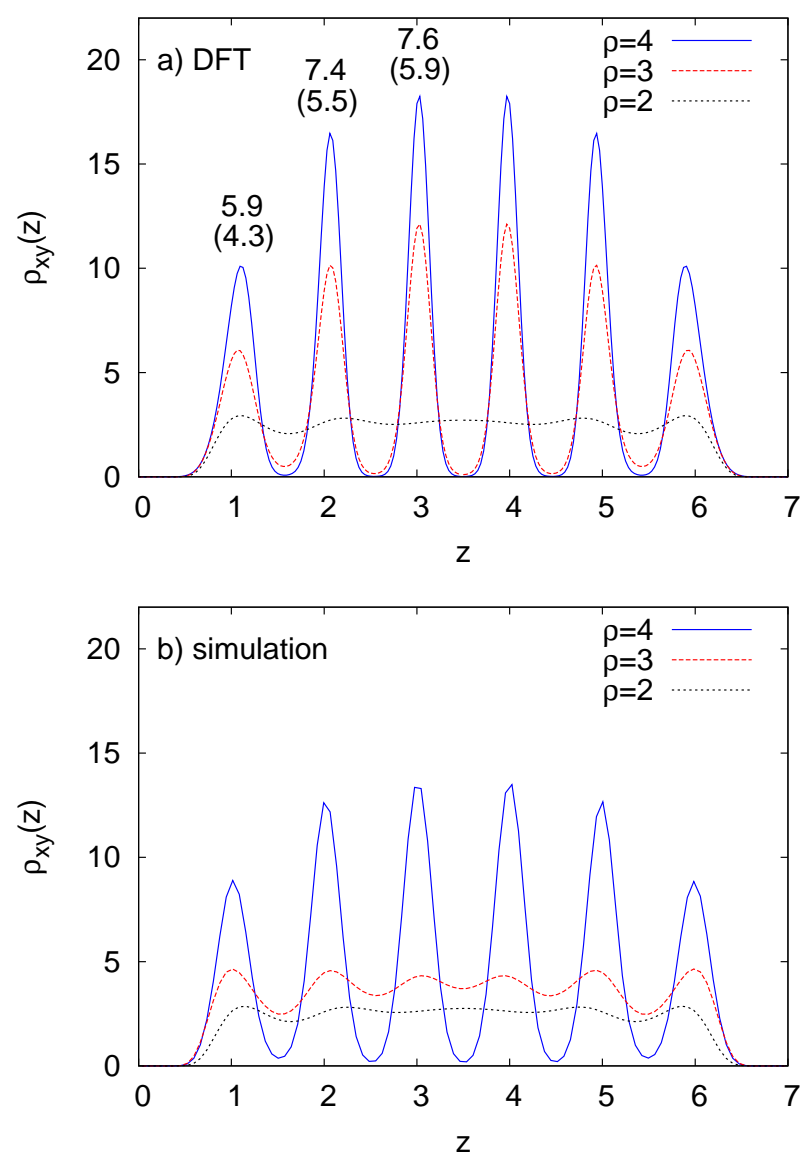

FIG. 4: Yukawa walls: Laterally-averaged density profiles $\rho_{x y}(z)$ for different average densities $(\rho=4,3,2$ from top to bottom) for $L_{z}=7$ as obtained from the DFT, (a), and the simulation, (b). (a) The fcc crystal in the DFT has the 100-orientation. The numbers over the peaks denote the average number of particles, $n_{c}$, occupying a cluster, for $\rho=4$ (top value) and $\rho=3$ (bracketed, lower value) from the DFT. (b) The lateral dimensions of the periodic simulation box are $L_{x}=L_{y}=20.43$.

find a particle close to the walls. Both freezing chemical potential and fluid/solid densities at coexistence display pronounced oscillations while approaching the respective bulk values with increasing $L_{z}$. This effect is clearly more pronounced for small wall-wall separations. After each spike of $\mu_{f}\left(L_{z}\right)$ in fig. 6, a new layer of crystalline clusters is introduced into the box. The local minima of $\mu_{f}\left(L_{z}\right)$ correspond to crystalline states with optimal layer spacing in the $z$-direction. Concomitantly, the wavelength of the oscillations equals the bulk value of the distance of the crystalline particle layers in the 111-direction, $c=1.04 c^{*}=1.113$ (cf. table II) line 4). This finding is qualitatively validated by the computer simulations (see fig. 7). Here, a simple criterion to separate crystalline from non-crystalline states was based on the value of the first minimum of the laterally averaged

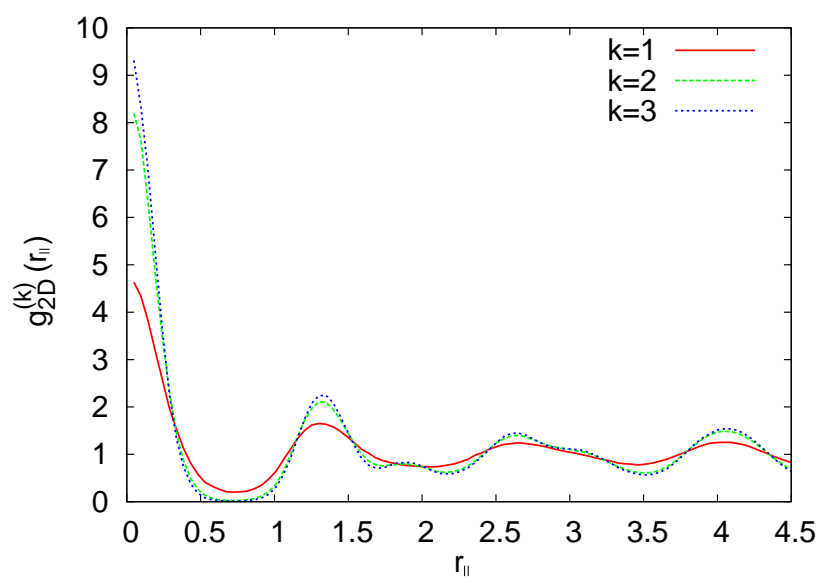

FIG. 5: Yukawa walls: The lateral pair distribution function $g_{2 \mathrm{D}}^{(k)}\left(r_{\|}\right)$for the first three layers of clusters on either of the wall ( $k$ is counted from the wall) for the same confinement as in fig. 4 at average density $\rho=4$ (for the definition of layers see main text).

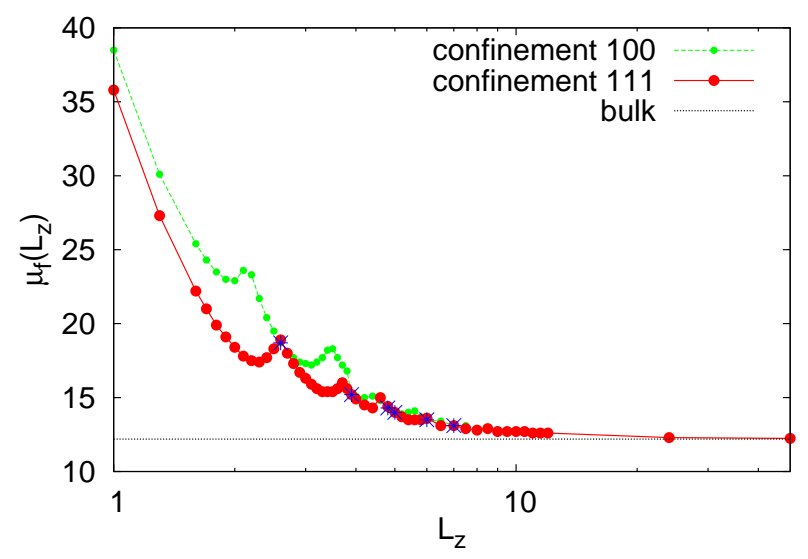

FIG. 6: Yukawa walls: Freezing chemical potential $\mu_{f}\left(L_{z}\right)$ as a function of the confinement width $L_{z}$ for the 111-orientation (red/solid) and the 100-orientation (green/dashed). The (blue) stars mark those points where the 100-orientation is stable versus the 111-orientation. The horizontal line displays the respective freezing value of the bulk system, $\mu_{f}=12.19$.

pair distribution functions $g_{2 \mathrm{D}}^{(k)}\left(r_{\|}\right)$of the most central layer $k$, which always freezes first among all layers. The simulation points in fig. 7 display the states of constant $\operatorname{minimum} g_{2 \mathrm{D}}^{(k)}\left(r_{\min }\right)=0.15$.

The confining walls have a profound influence on the grand potential of the system, especially for small values of $L_{z}$. Despite the lower average density within the slit pores, the grand potential density $\omega\left(\mu ; L_{z}\right) \equiv$ $\Omega\left[\rho_{\mathrm{eq}}\left(\mathbf{r} ; \mu, L_{z}\right)\right] / V$, plotted as a function of $\mu$ for different confinement lengths $L_{z}$ in fig. 8, is higher in confinement than in the bulk. In fig. 8, $\omega\left(\mu ; L_{z}\right)$ is represented both 


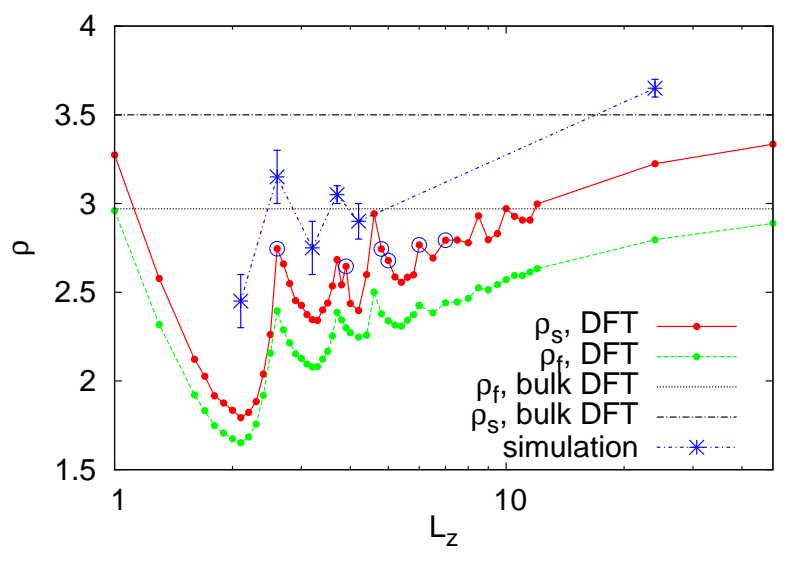

FIG. 7: Yukawa walls: Fluid and solid densities $\rho_{f}\left(L_{z}\right)$, $\rho_{s}\left(L_{z}\right)$ at coexistence. The open circles display the crystalline states which possess a 100-orientation with respect to the walls. Shown are also the respective bulk values. The DFT-results for the confined case are compared against the locus of constant $g_{2 \mathrm{D}}^{(k)}\left(r_{\min }\right)=0.15$ from the simulation, where $k$ refers to the most central layer (see main text).

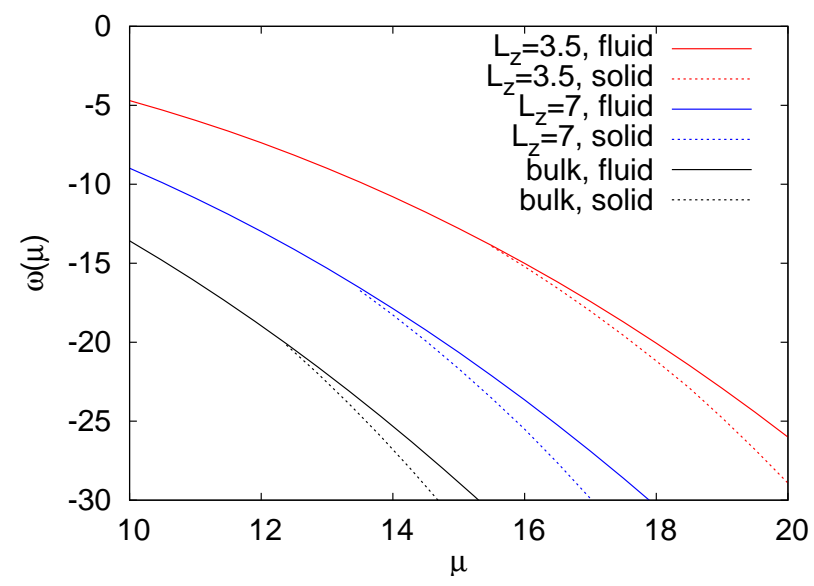

FIG. 8: Yukawa walls: Grand potential density $\omega(\mu)$ in the fluid (solid lines) and the solid state (dashed lines) as a function of chemical potential for $T=1$ for different confinement lengths $L_{z}=3.5,7$ and for the bulk (from top to bottom).

for the solid and the fluid state, the fluid being stable for $\mu<\mu_{f}\left(L_{z}\right)$ and metastable for $\mu>\mu_{f}\left(L_{z}\right)$.

For the case of small confinement lengths, $L_{z} \lesssim 7$, the favorite orientation of the compressed/expanded fcc crystal is, except within some tiny $L_{z}$-intervals, the 111orientation. As a consequence, the chemical potential at freezing for the 100-crystal which is metastable within the DFT, is almost always equal or higher than for the 111-crystal (cf. fig. 6), within the accuracy of the DFT calculation. This signifies that the system favors to have a layer of hexagonally crystalline clusters close to the

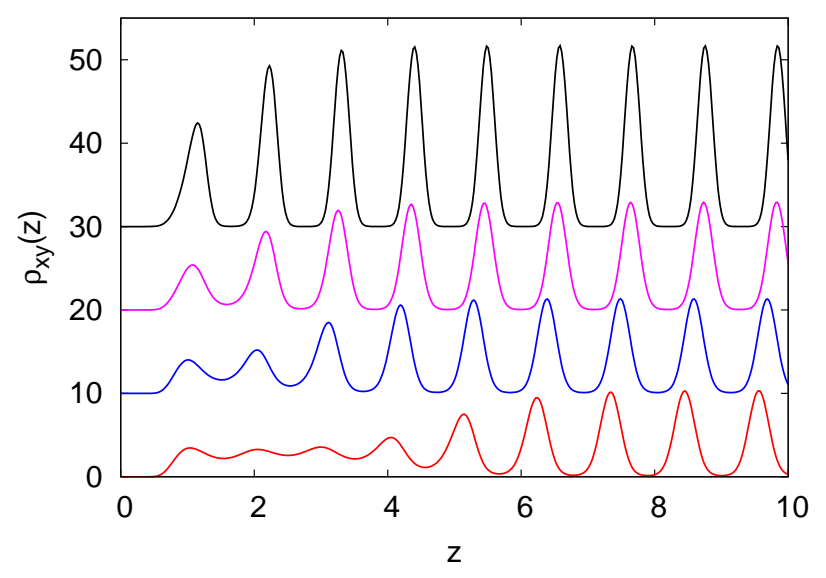

FIG. 9: Yukawa walls: Laterally-averaged density profiles $\rho_{x y}(z)$ close to the left wall for $L_{z}=48$ for different chemical potentials $\mu=16,13,12.5,12.24$ (from top to bottom).

walls, this layer being clearly visible in the simulation snapshots in fig. 11. We note exemplary for $L_{z}=7$, for which we find a stable 100-oriented crystal, that under consideration of the non-Bravais hep lattice, the latter is thermodynamically stable in comparison to the fcc one, albeit with a small difference in grand potential, leading again to a hexagonal cluster arrangement on the walls. The stability of the hcp versus the 100-oriented fcc lattice is also most likely to be found for the other confinement lengths but we did not check this in our study. For very large $L_{z}$, the difference in grand potential density between the 100- and the 111-orientations is decreasing and we focus on the 111-orientation for $L_{z} \geq 24$.

For large wall-wall separations, $L_{z} \gg 1$, as the bulk freezing chemical potential is approached from above $\left(\mu>\mu_{f}=12.19\right)$, the blurred clusters on the walls melt and a fluid layer grows on each wall. The walls enforce the growth of a liquid film, a property referred to as premelting. The number of molten layers $N_{m}$ on each wall increases continuously with decreasing difference in chemical potential $\Delta \mu \equiv \mu-\mu_{f}$, as can be seen from the $x y$-averaged density profiles in fig. 9 for the confinement length of $L_{z}=48$. In fig. 10, we show the number of molten layers on the walls as a function of $\Delta \mu$ for two different confinement lengths, $L_{z}=24$ and $L_{z}=48$. Here, a layer of what used to be crystalline clusters, is defined to be molten once the density within it exceeds the threshold value of $\rho(\mathbf{r})>0.15$ for all $x$ and $y$. This criterion has been used for the absence of knowledge about the pair-correlations, which was the basis for the simulation-results above. The $z$-position of a layer is defined by the $z$-position of the respective maximum of the $x$ - and $y$-averaged density field. After growing up to a maximum finite number of molten layers on each wall, $N_{m}^{c}\left(L_{z}\right)$, the system turns completely fluid due to capillary melting at $\mu_{f}\left(L_{z}\right)$, as is also visualized in fig. 10 by the black vertical bars. At the melting transition, the 


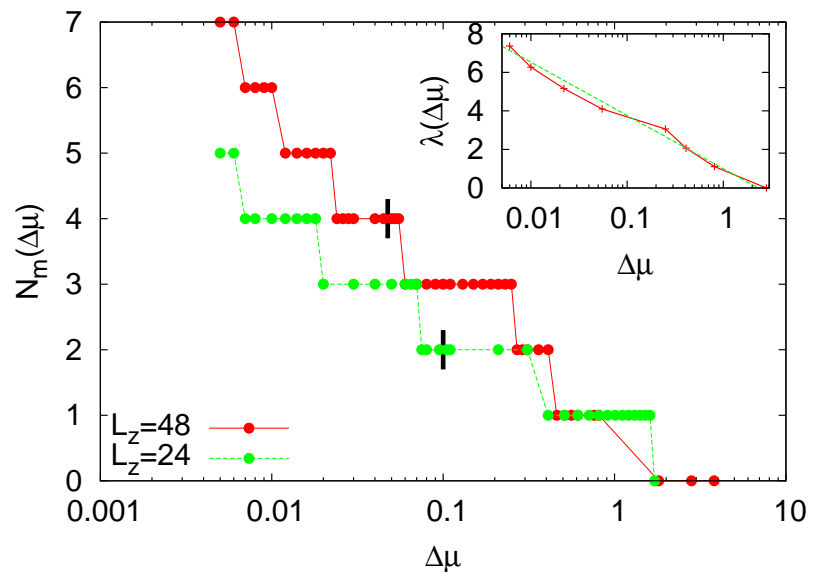

FIG. 10: Yukawa walls: The number of molten layers $N_{m}(\Delta \mu)$ on either Yukawa-wall as a function of the difference in chemical potential $\Delta \mu$. The black bars indicate the points of capillary melting, $\mu_{f}\left(L_{z}\right)$. The points to the left of the bars correspond to metastable crystalline states with the minimum grand potential among all possible crystalline states. We did display $N_{m}$ only for those chemical potentials for which at least one other still crystalline state with a higher number of molten layers was found to be metastable (see main text). Inset: The corresponding width $\lambda(\Delta \mu)$ of the fluid system close to the wall for $L_{z}=48$, evaluated at the kinks of $N_{m}(\Delta \mu)$. The dashed line is the least-square logarithmic fit $-\xi_{f} \ln (\Delta \mu)+$ const.

number of molten layers at each wall is still substantially smaller than the number of crystalline layers in the central region of the slit. In particular, we find for the maximum wall-wall-separation under study, $L_{z}=48$, that $N_{m}^{c}\left(L_{z}=48\right)=4$ which is still microscopic. For $L_{z}=24$ we find $N_{m}^{c}\left(L_{z}=24\right)=2$.

The occurrence of capillary filling when a system close to its bulk phase transition is confined between two parallel walls is a well-known phenomenon in the the context of liquid-gas phase coexistence of ordinary fluids [24, 47]. Here, we establish the existence of capillary melting from the walls for a system that forms crystalline layers in the middle of the slit pore. For the liquid-gas coexistence, capillary condensation precludes the wetting transition that takes place between two semi-infinite bulk phases [24. Wetting can be studied within DFT if one employs a semi-infinite system with the appropriate boundary condition at a distance $z \rightarrow \infty$ from the wall and it is straightforward to implement for the case of uniform phases 58. In our case, however, this would correspond to fixing a periodic crystal at $z \rightarrow \infty$, whilst the chemical potential $\mu$ is lowered towards its bulk value $\mu_{f}$ from above. This renders the DFT-calculation very complicated, therefore we will resort to a different approach in arguing that in the limit of infinite wall-wall separation, the fluid completely wets the Yukawa wall as $\mu \rightarrow \mu_{f}^{+}$. The approach is based on general, thermodynamic considerations that lead to scaling laws for the width of the wetting layer, which diverges at the bulk phase transition. Thus, the walls drive the system to the molten state.

In the limit of large wall-wall separation and for macroscopically thick fluid films of width $1 \ll \lambda(\Delta \mu) \ll L_{z}$ on either wall, the grand potential per unit surface area $A$ of the short-range interacting system can be written as [47, 59.

$$
\begin{array}{r}
\frac{\Omega(\Delta \mu)}{A}=2\left(\gamma_{f w}+\gamma_{f s}\right)+\omega\left(\mu_{f}\right) L_{z}+2 \epsilon_{1} \exp \left[-\frac{\lambda}{\xi_{f}}\right] \\
-\left[\left(L_{z}-2 \lambda\right) \rho_{s}+2 \lambda \rho_{f}\right] \Delta \mu+\mathrm{O}\left(\Delta \mu^{2}\right),
\end{array}
$$

where $\gamma_{f w}$ is the fluid-wall surface tension, $\gamma_{f s}$ is the fluid-solid surface tension, and $\epsilon_{1}$ is the prefactor of the interaction between the fluid-wall and the fluid-solid interfaces, which decays exponentially on the range of the correlation length of the fluid that wets the wall [47, 59. As thermodynamic quantities on the righthand side are evaluated at phase coexistence, use has been made of the identity $\partial \omega_{f, s} / \partial \mu=-\rho_{f, s}$, where $\omega_{f, s}$ are the grand potential densities of the fluid and the crystal at coexistence, respectively. The correlation length $\xi_{f}$ of the fluid is obtained as the inverse of the smallest imaginary part of the pole of the static structure factor $S(k)=\left[1-\rho_{f} \tilde{\phi}(k)\right]^{-1}$ closest to the real axis, i.e., $\xi_{f}=[\operatorname{Im}(k)]^{-1}=1.20$, where $k$ fulfills $1-\rho_{f} \tilde{\phi}(k)=0$ [58]. In the case of complete wetting, i.e., for

$$
\gamma_{s w}>\gamma_{f w}+\gamma_{f s}
$$

where $\gamma_{s w}$ is the wall-solid surface tension, two scaling behaviors follow from this ansatz. At first, upon approaching $\mu_{f}$ from above, the width of the fluid film $\lambda(\Delta \mu)$ grows logarithmically as a function of $\Delta \mu$ :

$$
\lambda(\Delta \mu) \simeq-\xi_{f} \ln (\Delta \mu)+\text { const },
$$

as long as $\lambda \ll L_{z}$ holds, i.e., as long as both walls behave each as single walls in contact with a semi-infinite crystal. Growth is indeed observed in our results but it is interrupted at the freezing transition $\mu_{f}\left(L_{z}\right)$ by capillary melting, which leads us to the second scaling relation: at the point of capillary melting, the net contribution of the fluid region in the middle of the slit pore to the grand potential per unit area, $\left(\omega_{f}-\omega_{s}\right)\left(L_{z}-2 \lambda\right)$ equals twice the liquid-solid surface tension $\gamma_{f s}$, which yields the following relation:

$$
L_{z}-2 \lambda\left(\mu_{f}\left(L_{z}\right)\right) \simeq \frac{2 \gamma_{f s}}{\left(\rho_{s}-\rho_{f}\right)\left(\mu_{f}\left(L_{z}\right)-\mu_{f}\right)} .
$$

For the maximum confinement-width studied $\left(L_{z}=48\right)$, we found $N_{m}^{c}=4$ at $\mu=\mu_{f}\left(L_{z}\right) \cong 12.24$ corresponding to $\lambda \cong 4.1$, which is too small to accurately be fitted to a logarithmic function and to extract $\mu_{f}\left(L_{z}\right)$. Nevertheless, within the DFT it is possible to avoid the transition to the stable, capillary-molten phase, and remain 
instead in a partly crystalline setup also for $\mu<\mu_{f}\left(L_{z}\right)$ by iterating eq. (5) with an appropriate initial density field $\rho(\mathbf{r})$, which already has a given number of molten layers. For a single wall, in which case capillary melting is absent, the system will choose the crystalline state with the lowest value of $\tilde{\Omega}[\rho(\mathbf{r})]$. Accordingly, we focus on the state that fulfills the same condition, among all metastable crystalline states, in the presence of two walls. However, we do consider only those chemical potentials for which at least one other state, which is still crystalline and has a higher number of molten layers, was found to be metastable, i.e., non-collapsed to a fluid. In this way, further growth of the fluid layer is observed up to a point where the crystalline slab in the middle of the slit becomes unstable towards collapse (see fig. 10). By performing a least-square fit of the whole growth (between $N_{m}=1$ and $N_{m}=6$ ) to the logarithmic growth of eq. (13), we obtain the bulk freezing chemical potential $\mu_{f}=12.19$ independently of the already known value from Section V] (see inset of fig. 10). In fact, the width $\lambda(\Delta \mu)$ was taken to be the distance of the most distant molten layer from the wall. As expected, for large numbers of molten layers we find $\lambda(\Delta \mu) \simeq c N_{m}(\Delta \mu)$, with the layer separation $c=1.04 c^{*}=1.113$, as discussed above. The independently obtained freezing chemical potential $\mu_{f}=12.19$ agrees perfectly with the freezing chemical potential obtained from the partly constrained bulk measurement in Section V. This finding constitutes a strong confirmation of the validity of the complete wetting scenario.

The second scaling relation, eq. (14), serves as a check, again for the largest confinement length $L_{z}=48$. The ingredient we are still missing is the surface tension $\gamma_{f s}$ at coexistence. The latter quantity can be obtained within DFT by comparing the grand potential $\Omega$ of a bulk system at $\mu=\mu_{f}$ with that of a system that contains stripes of equally sized liquid and crystalline parts that form two planar liquid-solid interfaces within the periodic 'box' of size $L_{x} \times L_{y} \times L_{z}$. We have performed this calculation using a box with the same $x$ - and $y$-dimensions as in the confining case and a longer $z$-length, $L_{z}=96$; clearly, there is no external potential in this case. In this way, we obtain $\gamma_{f s} \cong 0.49$, where the crystal is oriented in the 111-direction at the interface. Insertion of $\gamma_{f s}$, together with $\rho_{s}-\rho_{f}=0.56$ and $\mu_{f}\left(L_{z}=48\right)-\mu_{f}=0.05$ into eq. (14), yields $L_{z}-2 \lambda \cong 35.3$, which agrees reasonably well with $L_{z}-2 \lambda \cong 39.9$ from the direct comparison of the grand potential densities of the capillary-molten or crystalline states in confinement. It must be noted that we disregarded widths of the interfaces in our crude, 'sharpkink' treatment of the interfaces that led to eq. (14). In reality, the widths of the fluid-wall and of the fluid-solid interfaces are of the order of $\sim 2 \sigma$, which helps in explaining the discrepancy.

Capillary melting is a first-order phase transition, which comprises the possibility of non-equilibrium states close to or at the transition remaining metastable. In the DFT calculations, the metastability of non-molten

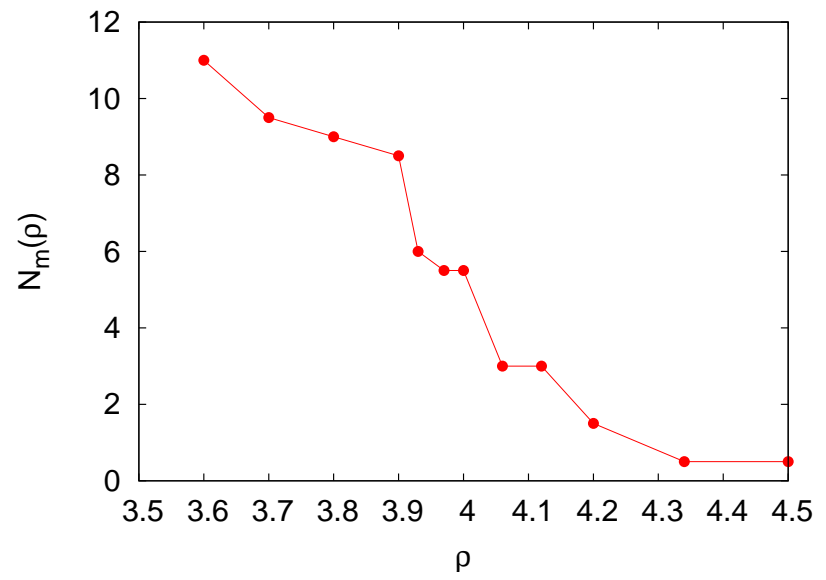

FIG. 11: Yukawa-walls: The average number of molten layers on each wall for the same confinement as in fig. 12 as a function of average density $\rho$, and as obtained from the computer simulations. The averages are performed over the left and the right wall.

states beyond equilibrium capillary melting has already been exploited for extracting the logarithmic growth of the wetting layer [see fig. 10 and eq. (13)]. In computer simulations of systems in the NVT-ensemble, metastable states may persist in small periodic simulation boxes and for average densities lying within two coexisting densities of a first-order phase transition. Here, free energy contributions of phase-separating interfaces are comparable or larger than bulk contributions [60. This behavior is also observed in computer simulations of the GEM-8 model in a relatively large planar confinement of $L_{z}=24$, where the lateral dimensions of the $x y$-periodic simulation box $L_{x}=L_{y}=9.29$ are comparatively small. Consequently, by continuously varying the density of the system a continuous growth of a liquid layer on each Yukawa wall up to complete filling of the box is observed (see fig. 11). Snapshots of the simulation are exemplarily shown for three different average densities $\rho=3.5,3.7,4.0$ in fig. 12 .

The average number of molten layers on each wall, $N_{m}(\rho)$ in fig. 11, has been determined by analyzing the lateral pair distribution function $g_{2 \mathrm{D}}\left(r_{\|}\right)$(not shown here) for a total of 22 crystalline layers in parallel to the walls, occurring for large densities. As for the narrow confinement of $L_{z}=7$ above, the range of each layer in the $z$-direction is determined by two consecutive minima of the respective density profile $\rho_{x y}(z)$ (also not shown here). A layer is defined to be molten/crystalline once the first minimum of $g_{2 \mathrm{D}}\left(r_{\|}\right)$is larger/smaller than 0.15 . Clearly, the system separates along the $z$-axis and does not show an indication of capillary melting. However, and as already argued above, we have strong evidence that the observation of states with a large number of molten layers is a finite-size effect in the simulations. 

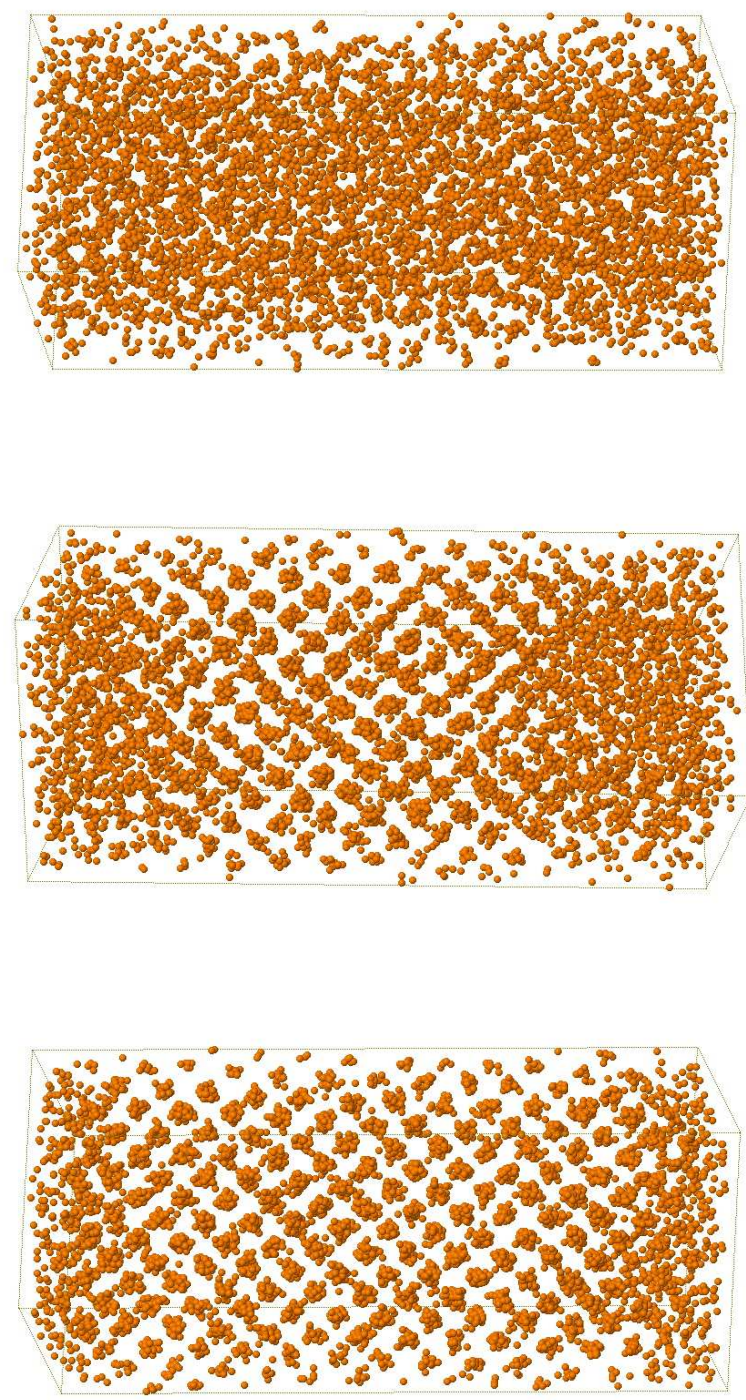

FIG. 12: Yukawa walls: Simulation snapshots for $L_{z}=24$ and average densities of $\rho=3.5,3.7,4.0$ (from top to bottom). The boundaries of the box $\left(L_{x}=L_{y}=9.29\right)$ are indicated by thin lines. The Yukawa-walls are situated at the left and at the right boundaries, respectively. The simulation boxes are oriented differently for better visibility of the crystalline region in the middle of the box.

\section{ATTRACTIVE WALLS}

In the second setup, we study the effect of attractive Lennard-Jones walls. The corresponding external potential is taken to have the form

$$
\begin{array}{r}
V_{\mathrm{LJ}}(\mathbf{r})=10\left[\frac{1}{z^{9}}-\frac{1}{z^{3}}+\frac{1}{\left(L_{z}-z\right)^{9}}-\frac{1}{\left(L_{z}-z\right)^{3}}\right], \\
0<z<L_{z} .
\end{array}
$$
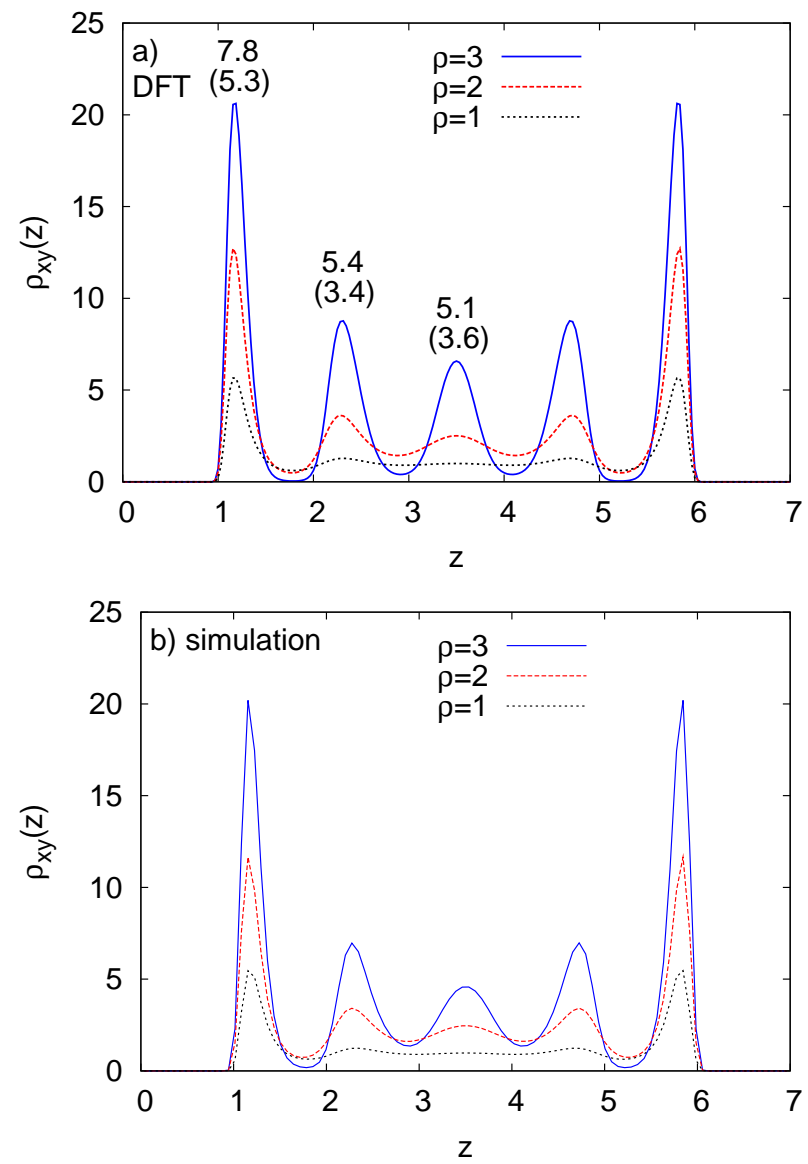

FIG. 13: Lennard-Jones walls: Laterally-averaged density profiles $\rho_{x y}(z)$ for three different densities above $(\rho=2,3)$ and below $(\rho=1)$ the freezing transition for $L_{z}=7$ as obtained from the DFT, (a), and the simulation, (b). The numbers over the peaks denote the average number of particles, $n_{c}$, occupying a cluster, for $\rho=3$ (top value) and $\rho=2$ (in parentheses, lower value). (b) The lateral dimensions of the periodic simulation box are $L_{x}=L_{y}=20.43$.

Recent numerical simulations by Lenz et al 46 have indeed shown that amphiphilic dendrimers of the second generation, demonstrated to be GEM-particles of the $Q^{ \pm}$-class in ref. [36, together with an attractive core wall-particle interaction lead to very similar effective interaction potentials. The precise shape of the wall potential depends, of course, on the different molecular interaction parameters. However, a range of the attraction of the order of $\sigma$ and a depth of the order of few $k_{B} T$ is certainly a reasonable assumption. Here, the minimum value of the Lennard-Jones potential for large $L_{z}$ is $V_{\mathrm{LJ}}\left(z_{\min } \cong 1.2\right) \cong-3.85$.

In contrast to the setup with repulsive, Yukawa walls of Section VI, the system now freezes at the walls before freezing in the middle region of the confinement, as can be seen for $L_{z}=7$ in fig. 13 . Note the big difference of cluster population close to the wall and in the 


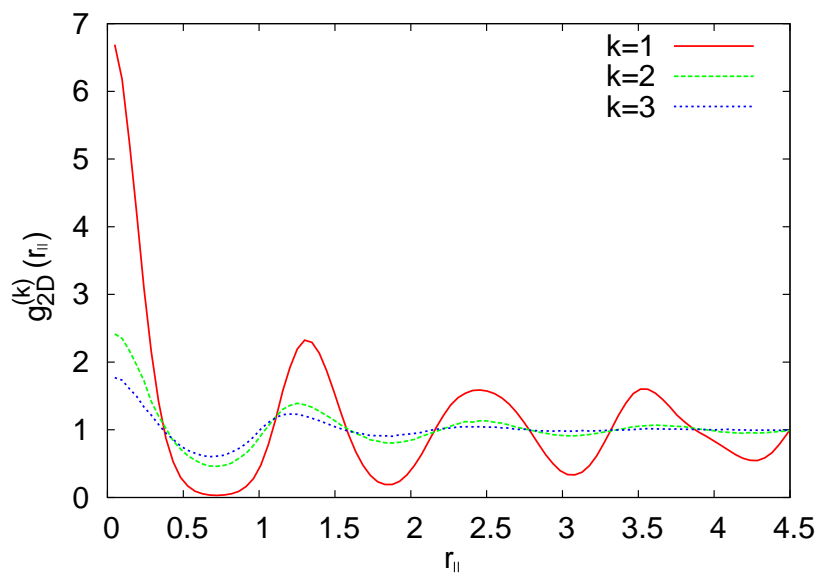

FIG. 14: Lennard-Jones walls: The lateral pair distribution function $g_{2 \mathrm{D}}^{(k)}\left(r_{\|}\right)$for the first three layers of clusters on either of the wall ( $k$ is counted from the wall) for the same confinement as in fig. 13 at average density $\rho=3$ (for the definition of layers see main text).

middle of the slit by more than 2 particles per cluster. As for the Yukawa setup, we present in fig. 13(b) results from BD computer simulations for the same parameters as in the DFT, which show the same quantitative behavior for $\rho=1$ and the same qualitative behavior for $\rho=2,3$. The reversal of the dependence of crystallinity on the layer-distance from the walls is confirmed by measurements of the restricted pair-distribution function, $g_{2 \mathrm{D}}^{(k)}\left(r_{\|}\right)$, eq. 10 , shown for the first three layers from either wall in fig. 14. Peaks and minima of the pair distribution function clearly become less pronounced for increasing distance of the layer from the wall.

Freezing in terms of breaking the $x y$-symmetry of the system is now mainly a single-wall effect, which is slightly disturbed by the interaction of the two crystalline layers on either wall with each other. This is at odds with freezing at the Yukawa setup, which is a bulk-dominated phenomenon disturbed by the walls. On these grounds, we denote the surface freezing chemical potential for the Lennard-Jones setup as $\mu_{f}^{s}\left(L_{z}\right)$, in order to distinguish it from the respective capillary freezing chemical potential $\mu_{f}\left(L_{z}\right)$ (see below). The bulk phase diagram only enters the stage once the bulk freezing chemical potential, $\mu_{f}$, is approached from below. Interactions between crystalline layers on either wall are subdominant for wall separations as small as $L_{z} \approx 5$. This can be ascertained from the dependence of the surface-freezing chemical potential $\mu_{f}^{s}\left(L_{z}\right)$ on $L_{z}$, fig. 15, which monotonically approaches a plateau value of $\mu_{f}^{s} \equiv \mu_{f}^{s}\left(L_{z} \rightarrow \infty\right)$ already from $L_{z}=5$ on. By extrapolating the phase diagram of fig. 15 to $L_{z} \rightarrow \infty$ we find $\mu_{f}^{s} \cong 7.7$ which corresponds to a bulk fluid density of $\rho \cong 1.90$. This value, of course, depends on the depth of the external potential.

Contrary to Yukawa walls, this setup shows capillary

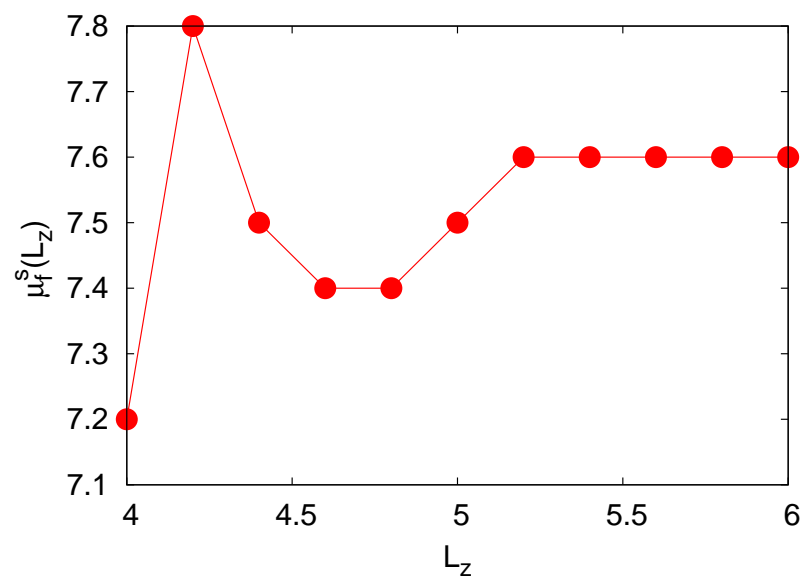

FIG. 15: Lennard-Jones walls: Surface freezing chemical potential $\mu_{f}^{s}\left(L_{z}\right)$ as a function of the confinement width $L_{z}$ for the attractive Lennard-Jones walls.

freezing from the walls. Whereas at $\mu_{f}^{s}\left(L_{z}\right)$ surfaceinduced freezing sets in, capillary freezing occurs at a significantly higher value $\mu_{f}\left(L_{z}\right)$, which now approaches $\mu_{f}$ from below as $L_{z}$ grows, i.e., $\mu_{f}\left(L_{z} \rightarrow \infty\right)=\mu_{f}$. We note also that freezing on the walls is not only observed for the prefactor 10 in eq. (15) but for any prefactor, i.e., for a vanishing attractive part of $V_{\mathrm{LJ}}(\mathbf{r})$, implying that surface-induced freezing is an intricate effect which sensitively depends on the nature of the wall-particle interaction. In particular, for the system under study the question whether surface-induced freezing or melting is observed, seems to be mostly a matter of the range or softness of the wall-particle potential. Our results suggest that there is surface-induced freezing for a hard wall (as for the Lennard-Jones walls) whereas there is surfaceinduced melting for a soft wall (as for the Yukawa-walls).

For small values of $L_{z}$, the two minima of the external potential merge and the system behaves qualitatively the same as for the repulsive Yukawa walls. We therefore restrict our analysis to $L_{z} \geq 4$. Due to the relatively strong attraction of the walls, the system favors a hexagonal pattern of clusters on the planar surfaces. The equilibrium orientation of the (distorted) fcc lattice in confinement is therefore always the 111-orientation. As the chemical potential is increased beyond $\mu_{f}^{s}\left(L_{z}\right)$ and as the bulk freezing chemical potential $\mu_{f}$ is approached from below, the number of crystalline layers on either wall grows continuously, as can be seen from the density profiles for $L_{z}=24$ in fig. 16 .

Upon approaching $\mu_{f}$ from below, we find for the Lennard-Jones walls that the number of crystalline layers on the walls, $N_{c}(\mu)$, grows continuously and nearly logarithmically with $|\Delta \mu|=\left|\mu-\mu_{f}\right|$ (cf. fig. 17 for $\left.L_{z}=24,48\right)$. This growth is eventually interrupted by capillary freezing, which occurs at a chemical potential $\mu_{f}\left(L_{z}\right)$. The surface- and bulk-freezing chemical potentials for attractive walls order as $\mu_{f}^{s}\left(L_{z}\right) \lesssim \mu_{f}^{s} \ll$ 


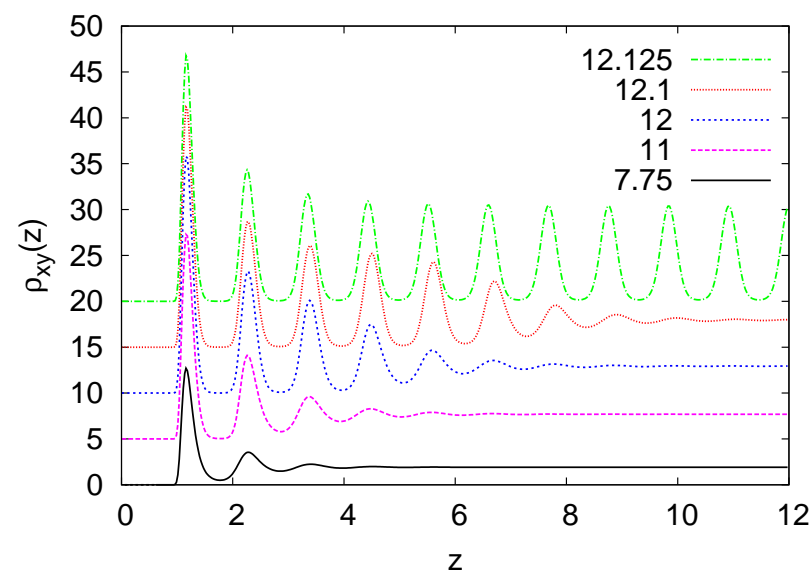

FIG. 16: Lennard-Jones walls: Laterally-averaged density profiles $\rho_{x y}(z)$ close to the left wall for $L_{z}=48$ for different chemical potentials $\mu=12.125,12.1,12,11,7.75$ (from top to bottom).

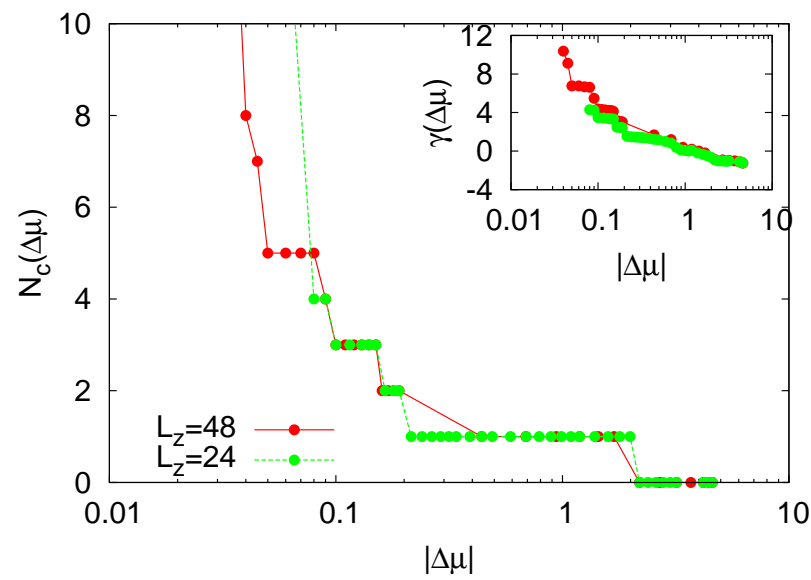

FIG. 17: Lennard-Jones walls: The number of crystalline layers on the walls, $N_{c}(\mu)$, as a function of the difference between the bulk freezing chemical potential, $\Delta \mu$ and for two different confinement widths. Note that $\mu_{f}>\mu$ here. Inset: the adsorption of a crystal layer $\gamma$, eq. 16 , as a function of $|\Delta \mu|$.

$\mu_{f}\left(L_{z}\right) \lesssim \mu_{f}$

As for the case of the Yukawa walls, we studied two large confinement lengths $L_{z}=24$ and $L_{z}=48$. We found $\mu_{f}\left(L_{z}=24\right)=12.12$ and $\mu_{f}\left(L_{z}=48\right)=12.16$. In the limit of large $L_{z}$ and large crystalline film thickness $\lambda$, still substantially smaller than $L_{z} / 2$, the (local) density of the fluid in the middle of the confining region is very close to the fluid density of a semi-infinite fluid reservoir on a single Lennard-Jones wall at infinity, which allows us to define the adsorption as

$$
\gamma=\int_{0}^{L_{z} / 2} \mathrm{~d} z\left[\rho_{x y}(z)-\rho_{x y}\left(L_{z} / 2\right)\right]
$$

which is plotted as a function of $|\Delta \mu|$ in the inset of fig. 17 .

Following the same reasoning as for the Yukawa walls, the slopes of the logarithmic growths of $N_{c}$ and $\gamma$ are now given by the correlation length of the (bulk) solid phase $\xi_{c}$ that wets the wall:

$$
\begin{aligned}
N_{c} & \simeq-\frac{\xi_{c}}{c} \ln (|\Delta \mu|)+\text { const }, \\
\gamma & \simeq-\left(\rho_{c}-\rho_{f}\right) \xi_{c} \ln (|\Delta \mu|)+\text { const },
\end{aligned}
$$

where $c=1.113$ is the distance of neighboring layers of the constrained bulk fcc crystal, again. By fitting a logarithmic curve to the $N_{c}$-data at intermediate $N_{c}$ we roughly obtain $\xi_{c} \cong 2$, roughly twice as large as the fluid correlation length $\xi_{f}$. As for the specular case of the Yukawa-walls, we cannot rule out the possibility of a non-continuous growth of the crystalline layer in a singlewall setup. However, our results for the two confinement widths of $L_{z}=24,48$ suggest that the crystal completely wets the Lennard-Jones walls and that the growth of the crystalline film is only interrupted by capillary freezing.

We present snapshots of BD-computer simulations of the system in planar Lennard-Jones confinement of $L_{z}=$ 24 for different average densities in fig. 18. For the smallest density of $\rho=2.5$ the system is only crystalline on the walls. For intermediate density, $\rho=4.0$, the system displays two equally large crystalline layers on both walls, and for $\rho=5.0$ the system turns completely crystalline. This behavior is again quantified through the analysis of $g_{2 \mathrm{D}}^{(k)}\left(r_{\|}\right)$for all crystalline/fluid layers parallel to the confining walls, as in Section VI. The resulting average number of crystalline layers on each wall, $N_{c}(\rho)$, is plotted as a function of average density in fig. 19 .

\section{CONCLUSIONS}

In conclusion, we studied the effect of repulsive and attractive slit-pore confinement on freezing and melting of cluster-forming, ultrasoft, repulsive particles of the GEM-8-class. For repulsive Yukawa-walls we found that the system freezes from the middle of the slit whereas for attractive Lennard-Jones walls it freezes at the walls before freezing in the middle. For small confinementwidths the respective freezing chemical potentials and liquid-solid coexistence densities display strong oscillations with varying slit width - the wavelength being equal to the distance of crystal layers in the bulk. However, whereas the oscillations are very pronounced for $L_{z} \lesssim 15$ in the case of the Yukawa-walls, they are damped already for $L_{z} \approx 5$ in the case of the Lennard-Jones walls. In the case of large confinement-widths we could find strong indications that upon approaching the bulk freezing chemical potential from above the Yukawa-walls are completely wetted by fluid whereas upon approaching the bulk freezing chemical potential from below the LennardJones walls are completely wetted by the crystal. 

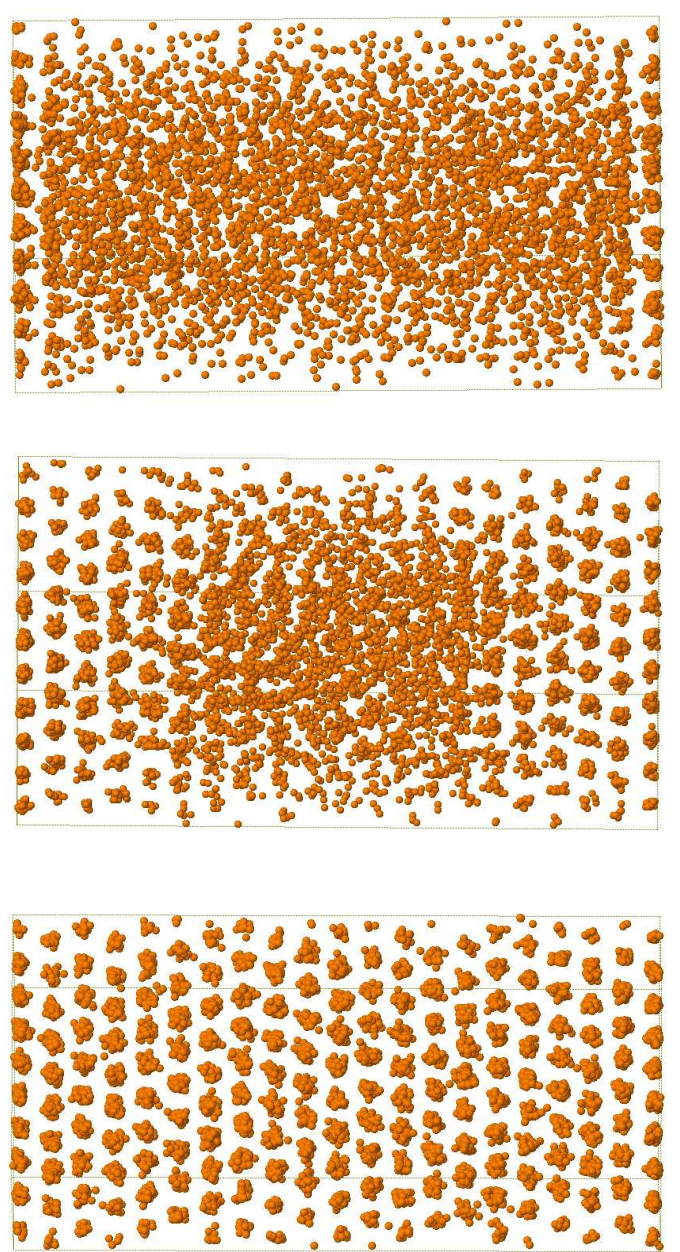

FIG. 18: Lennard-Jones walls: Simulation snapshots for $L_{z}=24$ and average densities $\rho=2.5,4.0,5.0$ (from top to bottom). The boundaries of the box $\left(L_{x}=L_{y}=9.29\right)$ are indicated by thin lines. The Lennard-Jones walls are situated at the left and at the right boundaries, respectively. The simulation boxes are oriented differently for better visibility of the crystalline region in the middle of the box.

Different modifications to the slit-pore geometry and to the particle-wall interactions are of interest: Whereas in this work both confining walls were of the same kind, it would be interesting to study the influence of "competing walls" 34. Further, the crystal arrangement at the walls is very sensitive towards structured/patterned substrates [61, 62, 63, 64. Moreover, slit pore confinement is a reference model for more complicated geometries such as porous media 65]. Confinement has also intriguing implications for the diffusive dynamics in and out of equilibrium. For equilibrium fluids in planar confinement, it has been recently demonstrated that both lateral and perpendicular diffusivities, $D_{\|}$and $D_{\perp}$, are enhanced at $z$-positions of higher average one-particle density $\rho_{x y}(z)$ as compared to the regions of lower den-

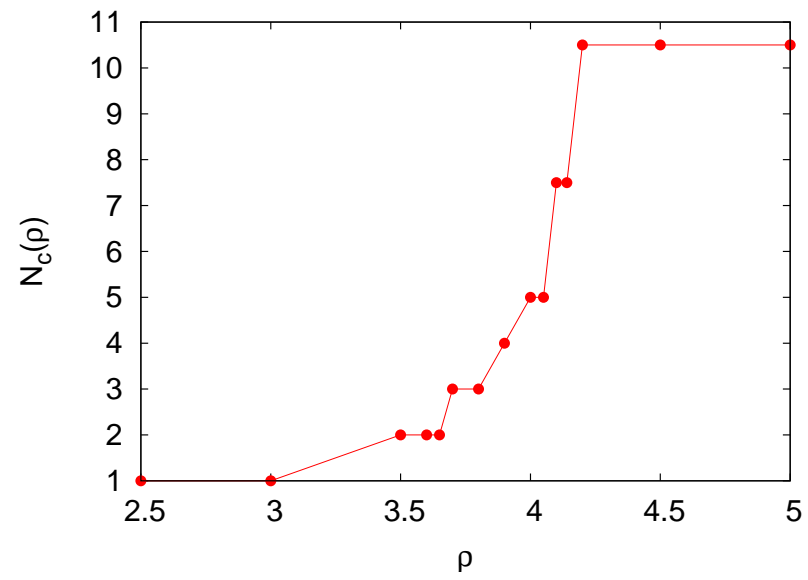

FIG. 19: Lennard-Jones-walls: The average number of crystalline layers on each wall for the same confinement as in fig. 18, as a function of average density $\rho$, and as obtained from the computer simulations. The averages are performed over the left and the right wall.

sity [66, 67]. For cluster-forming, GEM- $n$ particles, diffusive dynamics features novel characteristics, since even crystals now display nonzero long-time diffusivities, due to the presence of activated hopping processes [48, 49]. It would be interesting to examine the behavior of $D_{\|}$and $D_{\perp}$ in confinement, calculated separately for each layer, for the case at hand. Bulk studies [48, 49] have revealed an essentially Arrhenius-type behavior, $D \sim \exp \left(-\beta E_{A}\right)$, of the long-time diffusivity, with an activation energy $E_{A}$ proportional to $\rho$. It is interesting to find out to what extent this law holds also locally for each layer formed in slit-pore confinement. Recently, the relaxation dynamics of a fluid of Gaussian particles in a temporally oscillating, spherical, harmonic trap has been studied using computer simulations and dynamical density functional theory [68. The latter method is an extension of the DFT to overdamped non-equilibrium dynamics 69, 70, 71, which has recently also been used to study crystal growth in 2D [72. It would be interesting to employ dynamical density functional theory to study the relaxation of cluster crystals upon sudden or temporally periodic changes of the slit-pore confinement.

\section{Acknowledgments}

The authors would like to thank Hartmut Löwen and Bianca Mladek for helpful discussions. This work has been supported by the DFG within the SFB TR6, project section C3 and by the EU Network of Excellence "Softcomp". CNL wishes to thank the Erwin Schrödinger International Institute for Mathematical Physics (ESI, Vienna), where parts of this work have been carried out, for a Senior Research Fellowship and for its hospitality. AJM acknowledges support from 2007-60I201 (CSIC, Spain). 
[1] C. Alba-Simionesco, B. Coasne, G. Dosseh, G. Dudziak, K. Gubbins, R. Radhakrishnan, and M. SliwinskaBartkowiak, J. Phys.: Condens. Matter, 2006, 18, R15.

[2] L. D. Gelb, K. E. Gubbins, R. Radhakrishnan, and M. Sliwinska-Bartkowiak, Rep. Prog. Phys., 1999, 61, 1573.

[3] H. K. Christenson, J. Phys.: Condens. Matter, 2001, 13, R95.

[4] H. Löwen, Phys. Rep., 1994, 237, 249.

[5] J. E. Hug, F. van Swol, and C. F. Zukoski, Langmuir, 1995, 11, 111.

[6] D. J. Courtemanche and F. van Swol, Phys. Rev. Lett., 1992, 69, 2078 .

[7] D. J. Courtemanche, T. A. Pasmore, and F. van Swol, Mol. Phys., 1993, 80, 861.

[8] A. Fortini and M. Dijkstra, J. Phys.: Condens. Matter, 2006, 18, L371.

[9] M. Dijkstra, Phys. Rev. Lett., 2004, 93, 108303.

[10] Y. Hamada, K. Koga, and H. Tanaka, J. Chem. Phys., 2007, 127, 084908.

[11] E. M. Grzelak and J. R. Errington, J. Phys. C., 2008, 128, 014710 .

[12] R. Ohnesorge, H. Löwen, and H. Wagner, Phys. Rev. A, 1991, 43, 2870 .

[13] R. Ohnesorge, H. Löwen, and H. Wagner, Phys. Rev. E, 1994, 50, 4801.

[14] A. De Col, G. I. Menon, V. B. Geshkenbein, and B. G, Phys. Rev. Lett., 2006, 96, 177001.

[15] J. W. M. Frenken and J. F. van der Veen, Phys. Rev. Lett., 1985, 54, 134.

[16] A. van Blaaderen, MRS Bulletin, 2004, 29, 85.

[17] N. Osterman, D. Babič, I. Poberaj, J. Dobnikar, and P. Ziherl, Phys. Rev. Lett., 2007, 99, 248301.

[18] L. Salamacha, A. Patrikiejew, S. Sokolowski, and K. Binder, Eur. Phys. J. E, 2004, 13, 261.

[19] L. Salamacha, A. Patrikiejew, S. Sokolowski, and K. Binder, J. Chem. Phys., 2004, 120, 1017.

[20] R. Radhakrishnan, K. E. Gubbins, and M. SliwinskaBartkowiak, Phys. Rev. Lett., 2002, 89, 076101.

[21] X. Xu and S. Rice, Phys. Rev. E, 2008, 78, 011602.

[22] S. Auer and D. Frenkel, Phys. Rev. Lett., 2003, 91, 015703.

[23] R. Evans, Adv. Phys., 1979, 28, 143.

[24] R. Evans, J. Phys.: Condens. Matter, 1990, 2, 8989.

[25] D. W. Oxtoby, Nature, 1990, 347, 725.

[26] Y. Singh, Phys. Rep., 1991, 207, 351.

[27] J.-P. Hansen and I. R. McDonald, Theory of simple liquids, Academic Press, London, 2nd ed., 1986.

[28] T. V. Ramakrishnan and M. Yussouff, Phys. Rev. B, 1979, 19, 2775.

[29] D. W. Marr and A. P. Gast, Phys. Rev. E, 1993, 47, 1212.

[30] M. Schmidt and H. Löwen, Phys. Rev. E, 1997, 55, 7228.

[31] L. Mingqing, A. B. Michael, and M. F. David, J. Chem. Phys., 2007, 127, 164709.

[32] W. K. Kegel, J. Chem. Phys., 2001, 115, 6538.

[33] A. Imperio and L. Reatto, Phys. Rev. E, 2007, 76, 040402(R).

[34] K. Binder, J. Horbach, R. Vink, and A. De Virgiliis, Soft Matter, 2008, 4, 1555.

[35] C. N. Likos, Phys. Rep., 2001, 348, 267-349.
[36] B. M. Mladek, G. Kahl, and C. N. Likos, Phys. Rev. Lett., 2008, 100, 028301.

[37] A. Sütő, Phys. Rev. Lett., 2005, 95, 265501.

[38] A. Sütő, Phys. Rev. B, 2006, 74, 104117.

[39] S. Torquato and F. H. Stillinger, Phys. Rev. Lett., 2008, 100, 020602.

[40] C. N. Likos, A. Lang, M. Watzlawek, and H. Löwen, Phys. Rev. E, 2001, 63, 031206.

[41] C. N. Likos, B. M. Mladek, D. Gottwald, and G. Kahl, J. Chem. Phys., 2007, 126, 224502.

[42] B. M. Mladek, D. Gottwald, G. Kahl, M. Neumann, , and C. N. Likos, Phys. Rev. Lett., 2006, 96, 045701.

[43] B. M. Mladek, P. Charbonneau, and D. Frenkel, Phys. Rev. Lett., 2007, 99, 235702.

[44] A. Lang, C. N. Likos, M. Watzlawek, and H. Löwen, J. Phys.: Condens. Matter, 2000, 12, 5087.

[45] I. O. Götze, A. J. Archer, and C. N. Likos, J. Chem. Phys., 2006, 124, 084901.

[46] D. Lenz, R. Blaak, and C. N. Likos, unpublished.

[47] S. Dietrich in Phase Transitions and Critical Phenomena, ed. C. Domb and J. Lebowitz, Vol. 12; Academic Press, London, 1988; p. 1.

[48] A. J. Moreno and C. N. Likos, Phys. Rev. Lett., 2007, 99, 107801.

[49] C. N. Likos, B. M. Mladek, A. J. Moreno, D. Gottwald, and G. Kahl, Computer Physics Communications, 2008, 179, 71 .

[50] In fact, exact diagrammatic expansions show that $c(r)$ remains finite for all $r$, whereas diverging potentials do not at $r=0$. Thus, $c(r)=-\beta \phi(r)$ must break down in such case.

[51] M. P. Allen and D. J. Tildesley, Computer Simulation of Liquids, Clarendon Press, Oxford, 1987.

[52] D. L. Ermak, J. Chem. Phys., 1975, 62, 4189.

[53] S. van Teeffelen, unpublished.

[54] N. W. Ashcroft and N. D. Mermin, Solid State Physics, Holt, Rinehart and Winston, New York, 1976.

[55] By fixing the lattice constant to $a^{*}$ there is actually a stable bcc-phase intervening between the liquid-fcc-crystal phase transition, which is not persistent when relaxing the constrained of fixed lattice constant. We therefore ignore this subtlety here.

[56] Here and in the following, we denote the local minima of $\tilde{\Omega}[\rho(\mathbf{r})]$ with respect to the density field $\rho(\mathbf{r})$ by $\Omega[\rho(\mathbf{r})]$, although the grand potential is, of course, given by the minimum value of all local minima.

[57] B. M. Mladek, D. Gottwald, G. Kahl, M. Neumann, and C. N. Likos, J. Phys. Chem. B, 2007, 111, 12799.

[58] A. J. Archer, C. N. Likos, and R. Evans, J. Phys.: Condens. Matter, 2002, 14, 12031.

[59] T. Aukrust and E. Hauge, Physica A, 1987, 141, 427.

[60] Strictly speaking, these states are in equilibrium due to the (small) finite size but only for larger sizes they would be metastable.

[61] L. Harnau, F. Penna, and S. Dietrich, Phys. Rev. E, 2004, 70, 021505.

[62] H. Bohlen and M. Schoen, J. Chem. Phys., 2005, 123, 124714.

[63] M. Heni and H. Löwen, Phys. Rev. Lett., 2000, 85, 3668.

[64] M. Heni and H. Löwen, Phys. Rev. E, 2001, 13, 4675.

[65] A. Khokhlov, R. Valiullin, J. Kärger, F. Steinbach, and 
A. Feldhoff, New J. Phys., 2007, 9, 272.

[66] J. Mittal, T. M. Truskett, J. R. Errington, and G. Hummer, Phys. Rev. Lett., 2008, 100, 145901.

[67] G. Goel, W. P. Krekelberg, J. R. Errington, and T. M. Truskett, Phys. Rev. Lett., 2008, 100, 106001.

[68] M. Rex, C. N. Likos, H. Löwen, and J. Dzubiella, Mol. Phys., 2006, 104, 527.

[69] U. M. B. Marconi and P. Tarazona, J. Chem. Phys., 1999, 110, 8032.

[70] J. Dzubiella and C. N. Likos, J. Phys.: Condens. Matter, 2003, 15, L147.

[71] A. J. Archer and R. Evans, J. Chem. Phys., 2004, 121, 4246.

[72] S. van Teeffelen, C. N. Likos, and H. Löwen, Phys. Rev. Lett., 2008, 100, 108302. 